Further Progress in Noise Source Identification in High Speed Jets via Causality Principle

J. Panda

Ohio Aerospace Institute, Brook Park, Ohio

R.G. Seasholtz

Glenn Research Center, Cleveland, Ohio

K.A. Elam

Akima Corporation, Fairview Park, Ohio 
Since its founding, NASA has been dedicated to the advancement of aeronautics and space science. The NASA Scientific and Technical Information (STI) Program Office plays a key part in helping NASA maintain this important role.

The NASA STI Program Office is operated by Langley Research Center, the Lead Center for NASA's scientific and technical information. The NASA STI Program Office provides access to the NASA STI Database, the largest collection of aeronautical and space science STI in the world. The Program Office is also NASA's institutional mechanism for disseminating the results of its research and development activities. These results are published by NASA in the NASA STI Report Series, which includes the following report types:

- $\quad$ TECHNICAL PUBLICATION. Reports of completed research or a major significant phase of research that present the results of NASA programs and include extensive data or theoretical analysis. Includes compilations of significant scientific and technical data and information deemed to be of continuing reference value. NASA's counterpart of peerreviewed formal professional papers but has less stringent limitations on manuscript length and extent of graphic presentations.

- TECHNICAL MEMORANDUM. Scientific and technical findings that are preliminary or of specialized interest, e.g., quick release reports, working papers, and bibliographies that contain minimal annotation. Does not contain extensive analysis.

- CONTRACTOR REPORT. Scientific and technical findings by NASA-sponsored contractors and grantees.
- CONFERENCE PUBLICATION. Collected papers from scientific and technical conferences, symposia, seminars, or other meetings sponsored or cosponsored by NASA.

- SPECIAL PUBLICATION. Scientific, technical, or historical information from NASA programs, projects, and missions, often concerned with subjects having substantial public interest.

- TECHNICAL TRANSLATION. Englishlanguage translations of foreign scientific and technical material pertinent to NASA's mission.

Specialized services that complement the STI Program Office's diverse offerings include creating custom thesauri, building customized databases, organizing and publishing research results ... even providing videos.

For more information about the NASA STI Program Office, see the following:

- Access the NASA STI Program Home Page at http://www.sti.nasa.gov

- E-mail your question via the Internet to help@sti.nasa.gov

- Fax your question to the NASA Access Help Desk at 301-621-0134

- Telephone the NASA Access Help Desk at 301-621-0390

- Write to:

NASA Access Help Desk

NASA Center for AeroSpace Information 7121 Standard Drive

Hanover, MD 21076 
NASA/TM-2004-212385

AIAA-2003-3126

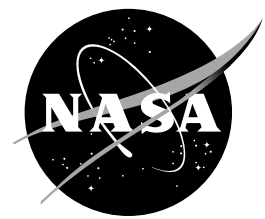

Further Progress in Noise Source Identification in High Speed Jets via Causality Principle

J. Panda

Ohio Aerospace Institute, Brook Park, Ohio

R.G. Seasholtz

Glenn Research Center, Cleveland, Ohio

K.A. Elam

Akima Corporation, Fairview Park, Ohio

Prepared for the

Nineth Aeroacoustics Conference and Exhibit cosponsored by the American Institute of Aeronautics and Astronautics and the Confederation of European Aerospace Societies

Hilton Head, South Carolina, May 12-14, 2003

National Aeronautics and

Space Administration

Glenn Research Center 


\section{Acknowledgments}

We acknowledge help from Amy F. Mielke, NASA Glenn Research Center, with data acquisition during a part of the experiment. The first author is thankful to Professor Philip J. Morris, Pennsylvania State University, and

Dr. Abbas Khavaran, QSS Group, Inc., for insightful discussions and useful suggestions on data analysis.

Trade names or manufacturers' names are used in this report for identification only. This usage does not constitute an official endorsement, either expressed or implied, by the National Aeronautics and Space Administration.

Available from

NASA Center for Aerospace Information 7121 Standard Drive

Hanover, MD 21076
National Technical Information Service 5285 Port Royal Road Springfield, VA 22100 


\title{
Further Progress in Noise Source Identification in High Speed Jets Via Causality Principle
}

\author{
J. Panda \\ Ohio Aerospace Institute \\ Brook Park, Ohio 44142 \\ R.G. Seasholtz \\ National Aeronautics and Space Administration \\ Glenn Research Center \\ Cleveland, Ohio 44135 \\ K.A. Elam \\ Akima Corporation \\ Fairview Park, Ohio 44126
}

\begin{abstract}
To locate noise sources in high-speed jets, the sound pressure fluctuations $\mathrm{p}^{\prime}$, measured at far field locations, were correlated with each of density $\rho$, axial velocity $u$, radial velocity $\mathrm{v}$, $\rho u u$ and $\rho v v$ fluctuations measured from various points in jet plumes. The experiments followed the cause-and-effect method of sound source identification, where the cross-correlation coefficients, $<\rho ; \mathrm{p}^{\prime}>,<\rho u u ; \mathrm{p}^{\prime}>$ etc., could be related to various source terms of Lighthill's equation. Detailed correlation surveys were conducted in three fully expanded, unheated plumes of Mach number $0.95,1.4$ and 1.8 . The velocity and density fluctuations were measured simultaneously using a recently developed, non-intrusive, point measurement technique based on molecular Rayleigh scattering (Seasholtz, Panda \& Elam, AIAA paper no 2002-0827). The technique uses a continuous wave, narrow line-width laser, Fabry-Perot interferometer and photon counting electronics. Light scattered by air molecules from a point on the laser beam was collected and spectrally resolved by a Fabry-Perot Interferometer. To determine Doppler shift caused by air flow, the image, formed after the interferometer, was split into two concentric parts and the intensity-ratio was measured by a pair of photomultiplier tubes. The change in the intensity ratio from that created by incident laser light provided a measure of a velocity component. Photo-electron counting over short-duration, contiguous bins provided a time history of velocity variation $\mathrm{u}(\mathrm{t}), \mathrm{v}(\mathrm{t})$. In addition, a part of the Rayleigh scattered light was measured directly, without passing through the interferometer, using a third photomultiplier tube to obtain a time history of density fluctuations $\rho(t)$; and finally, multiplications of the time series data provided $\rho u u(t)$ and $\rho v v(t)$. Two separate collection arrangements were used to measure Doppler shifts from $u$ and v velocity components. Fourier transforms of the time series data provided respective spectra. It was observed that the density spectra $S_{\rho}$ were in general similar to the axial velocity spectra while the radial velocity spectra $S_{v}$ were somewhat different. The $\rho-u$ cross-spectra show progressively decreasing correlation with increasing frequency. To determine sources of sound pressure fluctuations microphone
\end{abstract}

signals from 50 nozzle diameters and at polar angles from $30^{\circ}$ to $90^{\circ}$ to the jet axis, were cross-correlated with individual flow variables. The sound pressure fluctuations at $30^{\circ}$ to the jet axis provided the highest correlation coefficients with flow fluctuations. With an increase in microphone polar angle, the correlation coefficients decreased sharply, and beyond about $60^{\circ}$ all correlation mostly fell below the experimental noise floor. Among all turbulent fluctuations $\langle\rho u u ; p>$ correlations showed the highest values. Interestingly, $<\rho$; $\mathrm{p}^{\prime}>$, in all respects, were very similar to $\left\langle\rho \mathrm{puu} ; \mathrm{p}^{\prime}>\right.$. Both radial velocity $\mathrm{v}$ and $\rho \mathrm{v}$ fluctuations correlated very poorly, except for the $30^{\circ}$ microphone locations. By moving the laser probe at various locations in the jet it was found that the strongest noise source lies downstream of the end of the potential core and extends many diameters beyond. Correlation measurement from turbulent fluctuations along the lip shear layer showed a Mach number dependency. While significant correlations were measured in Mach 1.8 jet, values were mostly below the noise floor for subsonic Mach 0.95 jet. Various additional analyses, such as cross-spectra calculations, showed that fluctuations from large organized structures mostly contributed to the measured correlation, while that from small scale structures fell below the noise floor.

\section{INTRODUCTION}

The last six decades of research has not produced a unanimously accepted answer to the simple question of what produces noise from a jet flow. A vast number of earlier and current researchers have relied upon the acoustic analogy framework of Lighthill (1954), Lilley (1972) and others. Lighthill's equation is:

$$
\begin{gathered}
\frac{\partial^{2} \rho}{\partial \mathrm{t}^{2}}-a_{0}^{2} \nabla^{2} \rho=\frac{\partial^{2} \mathrm{~T}_{\mathrm{ij}}}{\partial \mathrm{X}_{\mathrm{i}} \partial \mathrm{X}_{\mathrm{j}}} ; \\
\mathrm{T}_{\mathrm{ij}}=\rho \mathrm{U}_{\mathrm{i}} \mathrm{U}_{\mathrm{j}}+\delta_{\mathrm{ij}}\left(\mathrm{p}-a_{0}^{2} \rho\right)
\end{gathered}
$$

where $\rho$ is air density, $p$ pressure, $\alpha_{0}$ ambient speed of sound, $U_{i}, U_{j}$ velocity vectors and $T_{i j}$ are the elements of the stress tensor. In the last few years there has been a steady growth of opposition to such an answer, based on multiple issues (Fedorchenko 2000, Tam 2001). A critical 
factor that has fueled the controversy is a lack of experimental verification of Lighthill sources, besides the $\mathrm{U}^{8}$ dependence of sound intensity, which only confirms proper dimensional scaling. Experimentally, thus far it has been impossible to directly measure the stress tensors. Even if one is able to measure all possible turbulence statistics, the next step: which parts of the turbulent fluctuations actually radiate in the far field, becomes even more difficult to determine. The common practice of using microphone arrays, elliptical and spherical mirrors in the far field to determine source location have both advantages and serious drawbacks. The advantage lies in the simplicity and the avoidance of measuring the complex turbulent flow, which is modeled simply as a distribution of monopoles. However, the noise sources are not monopoles and the presumed distribution functions required for data analysis have never been verified. Sound waves undergo a large amount of scattering before arriving at the far field; a linear extrapolation of sound path may lead to a wrong conclusion. There is a need to determine noise sources by independent means, which is the motivation for the present work. It can be argued that such an independent means can neither be achieved by only turbulence measurement/ simulation, nor by sole observations from far field, but through a simultaneous measurement of turbulent fluctuations (cause) and the far field noise (effect).

The direct correlation between the cause and effect was originally proposed by Siddon (see Siddon 1973, Rackl 1973) to locate sound sources, and during the 1970's many experimentalists had taken on the method. Usually, velocity (Lee \& Ribner 1972, Seiner \& Reethof 1974, Schaffer 1979, Richarz 1980 and others) or pressure fluctuations (Hurdle, Meecham \& Hodder 1974, Armstrong et al 1977 and others) in the jet were correlated with the sound pressure fluctuation measured by a fixed microphone. One attractive feature of this method is that the effects of scattering, absorption and refraction on sound radiation are automatically included by virtue of simultaneously extracting information from both the flow and acoustic fields. Another advantage in calculating the Lighthill source strength is that the fourth order derivative of the stress tensor is reduced to a second order one. Unfortunately, the promises were mostly unfulfilled in these earlier works, primarily due to a lack of non-intrusive turbulence measurement technique. The noise produced by intrusive hot-wire probes (Seiner 1974, and others) or microphones (Hurdle, Meecham \& Hodder, 1974 and others) placed inside the flow contributed to most of the correlation. Later on Laser Doppler Velocimetry (LDV) was used by Schaffer (1979) and Richarz (1980) among others. Schaffer wrote down the vast number of approximations and assumptions needed to relate the experimental data to theory. Nevertheless, the important issue of accuracy in velocity spectra measurements using LDV has lingered. The issues of seed particles following turbulent eddies, various biasing errors, and a limited dynamic range makes LDV a difficult tool to measure turbulence spectrum. An approximation, used by earlier researchers in evaluating Lighthill's stress tensors, is that the contribution from the density fluctuation is negligible: $\rho U_{\mathrm{i}} \mathrm{U}_{\mathrm{j}} \approx \bar{\rho} \mathrm{U}_{\mathrm{i}} \mathrm{U}_{\mathrm{j}}$, where $\bar{\rho}$ is time averaged density. Our recent work (Panda \& Seasholtz, 2002), on the other hand, shows that density fluctuations have significant correlation with far-field noise. It is to be noted that the role of density in Lighthill's formulation is ambivalent, every term in cause (right hand side of equation 1) and in effect (left hand side) contains a dependence on density.

Recently, a molecular Rayleigh scattering based technique has been advanced to simultaneously measure density and velocity fluctuations spectra in high-speed flow for the first time (Seasholtz, Panda \& Elam, 2001, 2002). Fluctuations occurring over frequency range of 0 to $50 \mathrm{kHz}$ have been measured. The technique is based on laser light scattering from the gas molecules present in air. Since neither any seed particles nor any intrusive probes are used, the technique is free from various problems faced by previous researchers. Air density fluctuation is the easiest to measure and has the highest accuracy. An in depth study of correlation between flow density fluctuations and sound pressure fluctuations from the peak noise emission direction had been reported earlier (Panda \& Seasholtz, 2002). Correlation study with the radial v velocity component and $\rho v v$ fluctuations had been reported by Panda, Seasholtz \& Elam (2000a). The present work is a continuation and banks on the additional capability of measuring axial component $(\mathrm{u})$ of velocity fluctuations.

Causality relation: Following is a recollection of the principles behind the Causality relation. The free space solution for Lighthill's wave equation for a field point $\mathbf{X}_{\mathbf{f}}$ from distributed source points $\mathbf{X}_{\mathbf{s}}$ (figure 1) is:

$$
\mathrm{p}^{\prime}\left(\mathbf{X}_{\mathbf{f}} ; \mathrm{t}\right)=\frac{1}{4 \pi} \frac{\partial^{2}}{\partial \mathrm{X}_{\mathrm{i}} \partial \mathrm{X}_{\mathrm{j}}} \int_{\mathbf{V}} \mathrm{T}_{\mathrm{ij}}\left(\mathbf{X}_{\mathbf{s}} ; \mathrm{t}^{\prime}\right) \frac{\mathrm{d}^{3} \mathbf{X}_{\mathbf{s}}}{\left|\mathbf{X}_{\mathbf{f}}-\mathbf{X}_{\mathbf{s}}\right|}
$$

Where, $p^{\prime}$ represents pressure fluctuations and the terms inside the integral are calculated at a retarded time to account for propagation from the source point to the field point: $\mathrm{t}^{\prime}=\mathrm{t}-\tau_{0}, \tau_{0}=\mid \mathbf{X}_{\mathbf{r}}-\mathbf{X}_{\mathbf{s}} / \alpha_{0}$. The integral is taken over the whole jet volume $\mathbf{V}$. Equation 1 shows that the stress tensor $T_{i j}$ has two terms. The first term $\frac{\partial^{2}\left(\rho U_{i} U_{j}\right)}{\partial X_{i} \partial X_{j}}$ has 9 different components, out of which the contribution from $\rho v v$ (v: radial component of velocity) and $\rho u u(u$ : axial component) fluctuations are measured in the present work. In the following, application of the causality relationship to the puu term is outlined first. Various 
issues involved in the interpretation are discussed next. Finally an extension of the causality relation to the second term of the stress tensor, $\left(p-\alpha_{0}^{2} \rho\right)$, is outlined. Following Proudman's analysis (1952), the double space divergence can be converted into double time derivative under two restrictions: (a) a scalar component of the stress tensor is measured along the direction of observation from the source, and (b) the field point is far enough to be in the radiation field of all sources. Under these conditions, the acoustic pressure at the field point can be written as:

$$
\mathrm{p}^{\prime}\left(\mathbf{X}_{\mathrm{f}} ; \mathrm{t}\right)=\frac{1}{4 \pi a_{0}^{2} \mathbf{r}} \int_{\mathrm{V}} \frac{\partial^{2}}{\partial \mathrm{t}^{\prime 2}}\left[\rho \mathrm{uu}\left(\mathbf{X}_{\mathrm{s}} ; \mathrm{t}^{\prime}\right)\right] \mathrm{d}^{3} \mathbf{X}_{\mathrm{s}}
$$

where $\mathbf{r}$ is radial distance. The scalar components of the stress tensor represent longitudinal quadrupoles, made by on-axis positioning of two opposite dipoles. Such a quadrupole has very strong directivity and, in the absence of convective amplification, the acoustic intensity falls as the $\cos ^{4}$ from the peak radiation direction. The peak radiation directions associated with

quadrupoles, $\frac{\partial^{2}(\rho \mathrm{uu})}{a_{0}^{2} \partial \mathrm{t}^{\prime 2}}$ and $\frac{\partial^{2}(\rho \mathrm{vv})}{a_{0}^{2} \partial \mathrm{t}^{\prime 2}}$, are respectively

along the jet axis and $90^{\circ}$ to the axis. The former is affected by refraction and convective amplification while the latter is not.

To obtain acoustic intensity an autocorrelation function for the above equation needs to be worked out. Usually, the auto-correlation function is created by multiplying space-time separated stress tensors separated in space by $\xi$ and in time by $\tau$.

$$
\begin{aligned}
& \left\langle\mathrm{p}^{\prime 2}\right\rangle\left(\mathbf{X}_{\mathrm{f}} ; \tau\right)=\frac{1}{16 \pi^{2} a_{0}^{4} \mathbf{r}^{2}} \\
& \int_{\xi} \int_{\mathbf{V}} \frac{\partial^{4}}{\partial \mathrm{t}^{4}}\left\langle\rho u u\left(\mathbf{X}_{\mathrm{s}} ; \mathrm{t}^{\prime}\right) \rho u u\left(\mathbf{X}_{\mathrm{s}}+\boldsymbol{\xi} ; \mathrm{t}^{\prime}+\tau\right)\right\rangle \mathrm{d}^{3} \mathbf{X}_{\mathrm{s}} \mathrm{d} \boldsymbol{\xi}
\end{aligned}
$$

In the "causality" relationship, however; this is accomplished by multiplying the source integral with the far field sound pressure:

$$
\begin{aligned}
& \left\langle\mathrm{p}^{\prime 2}\right\rangle\left(\mathbf{X}_{\mathrm{f}} ; \tau\right)= \\
& \left\langle\left[\frac{1}{4 \pi a_{0}^{2} \mathbf{r}} \int_{\mathrm{v}} \frac{\partial^{2}}{\partial \mathrm{t}^{\prime 2}}\left[\rho \mathrm{uu}\left(\mathbf{X}_{\mathrm{s}} ; \mathrm{t}^{\prime}+\tau\right)\right] \mathrm{d}^{3} \mathbf{X}_{\mathrm{s}}\right]\left[\mathrm{p}^{\prime}\left(\mathbf{X}_{\mathrm{f}} ; \mathrm{t}\right)\right]\right\rangle \\
& \quad=\frac{1}{4 \pi \mathbf{r} a_{0}^{2}} \int_{\mathbf{V}} \frac{\partial^{2}}{\partial \tau^{2}}\left[\mathrm{R}_{\rho \mathrm{\rho uu} ; \mathrm{p}^{\prime}}\left(\mathbf{X}_{\mathrm{s}}, \mathbf{X}_{\mathrm{f}} ; \tau\right)\right] \mathrm{d}^{3} \mathbf{X}_{\mathrm{s}} \\
& \mathrm{R}_{\rho \mathrm{\rho uu} ; \mathrm{p}^{\prime}}\left(\mathbf{X}_{\mathrm{s}}, \mathbf{X}_{\mathrm{f}} ; \tau\right)=\left\langle\rho \mathrm{puu}\left(\mathbf{X}_{\mathrm{s}} ; \mathrm{t}+\tau-\tau_{0}\right) \mathrm{p}^{\prime}\left(\mathbf{X}_{\mathrm{f}} ; \mathrm{t}\right)\right\rangle
\end{aligned}
$$

The $<>$ bracketed expressions imply time averages. Also, the correlation function $\mathrm{R}_{\text {puu; }}$, has to be calculated after shifting the puu data by the propagation time $\tau=-\tau_{0}$, or inversely the pressure fluctuation data by $\tau=\tau_{0}$. In essence, equation 5 expresses the radiated acoustic field in terms of a time delayed integral taken over the entire source volume, and consists of correlation functions between far field sound pressure p' and source field puu.

It is interesting to point out a particular advantage of equation 5 over 4 . The Lighthill's equation by itself is unable to separate hydrodynamic and acoustic

fluctuations. The former is the pressure field associated with turbulent fluctuations, and the latter is the radiated fluctuations that propagate far away from the turbulent motion. Lighthill's equation is nothing but a reformulation of the mass and momentum conservation equations and therefore every solution upholds these two physical laws. It is known that only a small part of the disturbances created by turbulent motion inside a jet ultimately radiates as sound. Since both radiating and non-radiating disturbances satisfy the same conservation laws, Lighthill's equation is unable to separate the two. Additional criteria are necessary, such as a frequencywave number, $\omega-\kappa$, analysis of the turbulent motion and imposition of a condition of supersonic convective speed with respect to the ambient sound speed: $\omega / \kappa \geq \alpha_{0}$. The traditional auto-correlation function in equation 4 is created by correlating two source points. In order to determine which part of this correlation ultimately radiates as sound, an additional wave number - frequency analysis of the right hand side is required (Morris et al 2002, Goldstein, 1976). On the other hand, multiplication by the field point in equation 5 , effectively imposes a filter function, since by definition a microphone kept in a far-field location only senses the radiated part of disturbances. Therefore, the cross-correlation function $\mathrm{R}_{\text {puu; }}$, separates out hydrodynamic and acoustic fluctuations. There is no need to perform additional frequency-wave number analysis.

The causality relationship requires a second timederivative, which ought to be avoided in experiments. Fourier transform of equation 5 yields (noting that the autocorrelation function is transformed to power spectral density and cross-correlation to cross-spectrum):

$$
\begin{aligned}
& \mathrm{S}_{\mathrm{p}^{\prime 2}}\left(\mathbf{X}_{\mathrm{f}} ; \mathrm{f}\right) \mathrm{df}=-\frac{\pi \mathrm{f}^{2} \mathrm{df}}{\mathbf{r} a_{0}^{2}} \int_{\mathrm{V}} \mathrm{S}_{\rho \mathrm{uu}, \mathrm{p}^{\prime}}\left(\mathbf{X}_{\mathrm{f}}, \mathbf{X}_{\mathrm{s}} ; \mathrm{f}\right) \mathrm{d}^{3} \mathbf{X}_{\mathrm{s}} \\
& \text { cross-spectrum : } \mathrm{S}_{\rho \mathrm{puu}, \mathrm{p}^{\prime}}\left(\mathbf{X}_{\mathrm{f}}, \mathbf{X}_{\mathrm{s}} ; \mathrm{f}\right)= \\
& \int_{-\infty}^{\infty} \mathrm{R}_{\rho \mathrm{\rho uu} ; \mathrm{p}^{\prime}}\left(\mathbf{X}_{\mathrm{s}}, \mathbf{X}_{\mathrm{f}} ; \tau\right) \exp (-\mathrm{j} 2 \pi \mathrm{f} \tau) \mathrm{d} \tau
\end{aligned}
$$

Finally, the acoustic intensity at the field point $\mathbf{X}_{\mathbf{f}}$ :

$$
\begin{aligned}
& \mathrm{I}\left(\mathbf{X}_{\mathbf{f}}\right)=\frac{1}{a_{0} \rho_{0}} \int_{-\infty}^{\infty} \mathrm{S}_{\mathrm{p}^{\prime 2}} \mathrm{df} \\
& =-\frac{\pi}{\mathrm{r} \rho_{0} a_{0}^{3}} \int_{-\infty \mathrm{V}}^{\infty} \int_{\mathrm{f}^{2}} \mathrm{~S}_{\text {puu, } \mathrm{p}^{\prime}}\left(\mathrm{X}_{\mathrm{f}}, \mathrm{X}_{\mathrm{s}} ; \mathrm{f}\right) \mathrm{d}^{3} \mathrm{X}_{\mathrm{s}} \mathrm{df}
\end{aligned}
$$


The above equation shows that the intensity of sound radiation is directly dependent on the distribution of cross-spectrum function in the flow field.

There are multiple issues surrounding the causality method. At a first glance the right hand sides of equations $4 \& 5$ are expected to be equal although the integrands are different. However, they may not be so as the difference between propagating and non-propagating disturbances is included in the latter. There is an issue of non-uniqueness in the application of causality method (Ffowcs Williams 1973). Since the sound pressure at the field point is a large sum over the entire sound-producing region of the jet, an unlimited number of variations in the source correlation can lead to the same summation at the field point. This criticism is not special for the causality technique but in general true for the more common form of source description via two-source points correlation (eqn. 4), which likewise, has to be integrated over the source volume. In a broader sense, many inverse problems in physics are of this nature. Although mathematically this appears to be a problem, it can be argued that the distribution of correlation functions measured in a real experiment is the valid distribution.

The interpretation of the correlation function is another issue that brings back a long-standing discussion of whether turbulence consists of uncorrelated small eddies or large vortical structures of significant spatial coherence. If turbulence is described by the former then the $<\rho u u, p>$ correlation function measured from various points in the jet are mutually independent; the integration over the jet volume becomes a simple addition in power and the correlation data can be used to determine source efficiency along the jet axis (Seiner \& Reethof, 1974). Such an interpretation also leads to a discussion of the number of eddies responsible for sound generation at a given instant (Lee \& Ribner, 1972). It is now well established from various experimental observations that low Strouhal frequency turbulent fluctuations are dominated by organized vertical waves with significantly long spatial coherence. The effect of source-coherence on jet noise has been discussed by Michalke (1983). For sources with long coherence length scales the phase of $<$ puu,p $>$ correlation will vary from point to point and the integration over the jet volume requires a knowledge of both magnitude and phase.

So far discussions have been confined to the contribution from the puu term only. It is straightforward to extend this analysis to include the second term of $\mathrm{T}_{\mathrm{ij}}$ tensor, equation 1 . The far field pressure fluctuations caused by pressure and density fluctuations in the plume are:

$$
\begin{aligned}
& \mathrm{p}^{\prime}\left(\mathbf{X}_{\mathbf{f}} ; \mathrm{t}\right) \\
& =\frac{1}{4 \pi a_{0}^{2} \mathbf{r}} \int_{\mathbf{V}} \frac{\partial^{2}}{\partial \mathrm{t}^{\prime 2}}\left[\left\{\mathrm{p}^{\prime}-a_{0}^{2} \rho^{\prime}\right\}\left(\mathbf{X}_{\mathrm{s}} ; \mathrm{t}^{\prime}\right)\right] \mathrm{d}^{3} \mathbf{X}_{\mathrm{s}}
\end{aligned}
$$

To completely evaluate this term one needs to measure at least temperature and density fluctuations inside the plume. The present experimental setup is unable to do the former. Neglecting contribution from entropy fluctuations, local sound speed $\mathrm{a}_{\mathrm{s}}$ relates pressure fluctuations to density fluctuations: $\mathrm{p}^{\prime}=\alpha_{\mathrm{s}}^{2} \rho^{\prime}$. Since $\alpha_{\mathrm{s}}^{2} /$ $\alpha_{0}^{2} \approx T_{j} / T_{0}$, where $T_{j}$ is jet temperature and $T_{0}$ is ambient temperature, the above equation becomes:

$$
\begin{aligned}
& p^{\prime}\left(\mathbf{X}_{\mathrm{f}} ; \mathrm{t}\right) \\
& =\frac{1}{4 \pi \mathbf{r}} \int_{\mathbf{v}} \frac{\partial^{2}}{\partial \mathrm{t}^{\prime 2}}\left[\left\{\frac{\mathrm{T}_{\mathrm{j}}}{\mathrm{T}_{0}}-1\right\} \rho^{\prime}\left(\mathbf{X}_{\mathrm{s}} ; \mathrm{t}^{\prime}\right)\right] \mathrm{d}^{3} \mathbf{X}_{\mathrm{s}} .
\end{aligned}
$$

where $\mathrm{T}_{0}$ is the ambient temperature. For an unheated low Mach number jet $\mathrm{T}_{\mathrm{s}} / \mathrm{T}_{0} \approx 1$ and the right hand side has negligible contribution to the far field. However, as the Mach number $M$ increases the effectiveness of this term is expected to increase as $\mathrm{M}^{2}$. A reasonable estimate may be obtained by using time average plume temperature:

$$
p^{\prime}\left(\mathbf{X}_{\mathbf{f}} ; t\right)=\frac{\left(\frac{\mathrm{T}_{j}}{\mathrm{~T}_{0}}-1\right)}{4 \pi \mathbf{r}} \int_{\mathbf{V}} \frac{\partial^{2}}{\partial \mathrm{t}^{\prime 2}}\left[\rho^{\prime}\left(\mathbf{X}_{\mathbf{s}} ; \mathrm{t}^{\prime}\right)\right] \mathrm{d}^{3} \mathbf{X}_{\mathbf{s}}
$$

Once again, multiplying the source integral with the far field pressure fluctuations:

$$
\begin{aligned}
& \left\langle\mathrm{p}^{\prime 2}\left(\mathbf{X}_{\mathrm{f}} ; \tau\right)\right\rangle \\
& =\frac{\left(\frac{\mathrm{Tj}}{\mathrm{T}_{0}}-1\right)}{4 \pi \mathbf{r}} \int_{\mathbf{V}} \frac{\partial^{2}}{\partial \tau^{2}}\left[\mathrm{R}_{\rho ; \mathrm{p}^{\prime}}\left(\mathbf{X}_{\mathrm{s}}, \mathbf{X}_{\mathrm{f}} ; \tau\right)\right] \mathrm{d}^{3} \mathbf{X}_{\mathrm{s}} \\
& \text { where, } \mathrm{R}_{\rho ; \mathrm{p}^{\prime}}\left(\mathbf{X}_{\mathrm{s}}, \mathbf{X}_{\mathrm{f}} ; \tau\right) \\
& =\left\langle\rho^{\prime}\left(\mathbf{X}_{\mathrm{s}} ; \mathrm{t}+\tau-\tau_{0}\right) \mathrm{p}^{\prime}\left(\mathbf{X}_{\mathrm{f}} ; \mathrm{t}\right)\right\rangle
\end{aligned}
$$

Since data analysis is performed in narrowband frequencies, Fourier transform of above yields: $\mathrm{S}_{\mathrm{p}^{\prime 2}}\left(\mathbf{X}_{\mathbf{f}} ; f\right) \mathrm{df}$

$=\left(1-\frac{\mathrm{T}_{\mathrm{j}}}{\mathrm{T}_{0}}\right) \frac{\pi \mathrm{f}^{2} \mathrm{df}}{\mathbf{r}} \int_{\mathrm{V}} \mathrm{S}_{\rho, \mathrm{p}^{\prime}}\left(\mathbf{X}_{\mathrm{f}}, \mathbf{X}_{\mathrm{s}} ; \mathrm{f}\right) \mathrm{d}^{3} \mathbf{X}_{\mathrm{s}}$

$$
\begin{aligned}
& \text { cross-spectrum : } S_{\rho, p^{\prime}}\left(\mathbf{X}_{\mathbf{f}}, \mathbf{X}_{\mathbf{s}} ; f\right) \\
& =\int_{-\infty}^{\infty} R_{\rho ; p^{\prime}}\left(\mathbf{X}_{s}, \mathbf{X}_{\mathbf{f}} ; \tau\right) \exp (-j 2 \pi f \tau) d \tau
\end{aligned}
$$

Therefore, a correlation between the flow density fluctuations and the far field sound pressure fluctuations provides an estimate of contribution from the second term of Lighthill's stress tensor. 
It is best to present the experimental data in a non-dimensionalized form. Following the traditional acoustic analogy approach, two different velocity scales are applied: ambient sound speed $\alpha_{0}$ for field points and $\mathrm{U}_{\mathrm{j}}$ for source points. The length and time scales are derived using the jet diameter D. Ambient value $\rho_{0}$ is used to normalize density. Using superscript * for nondimensionalized quantities, $S_{t}$ for Strouhal number, and $\mathrm{M}_{\mathrm{a}}$ for Mach number based on ambient sound speed, equation 6 becomes:

$$
\begin{aligned}
& \mathrm{S}_{\mathrm{p}^{\prime 2}}^{*}\left(\mathbf{X}_{\mathrm{f}}^{*} ; \mathrm{S}_{\mathrm{t}}\right) \mathrm{d} \mathrm{S}_{\mathrm{t}} \\
& =-\mathrm{M}_{\mathrm{a}}^{4} \frac{\pi \mathrm{S}_{\mathrm{t}}^{2}}{\mathrm{r}^{*}} \mathrm{dS}_{\mathrm{t}} \int_{\mathrm{V}} \mathrm{S}_{\rho \mathrm{puu}, \mathrm{p}^{\prime}}^{*}\left(\mathbf{X}_{\mathrm{f}}^{*}, \mathbf{X}_{\mathrm{s}}^{*} ; \mathrm{S}_{\mathrm{t}}\right) \mathrm{d}^{3} \mathbf{X}_{\mathrm{s}}^{*}, \\
& \mathrm{~S}_{\mathrm{t}}=\frac{\mathrm{fD}}{\mathrm{U}_{\mathrm{j}}}, \quad \mathrm{M}_{\mathrm{a}}=\frac{\mathrm{U}_{\mathrm{j}}}{a_{0}}, \quad \mathrm{~S}_{\mathrm{p}^{\prime 2}}^{*}=\frac{\mathrm{S}_{\mathrm{p}^{\prime 2}}}{\left(\rho_{0} a_{0}^{2}\right)^{2}}, \\
& \mathrm{~S}_{\rho \mathrm{\rho uu}, \mathrm{p}^{\prime}}^{*}=\frac{\mathrm{S}_{\rho \mathrm{puu}, \mathrm{p}^{\prime}}}{\left(\rho_{0} \mathrm{U}_{\mathrm{j}}^{2}\right)\left(\rho_{0} a_{0}^{2}\right)}
\end{aligned}
$$

Similarly, non-dimensionalized contribution from the second term (equation 12) is:

$$
\begin{aligned}
& \mathrm{S}_{\mathrm{p}^{\prime 2}}^{*}\left(\mathbf{X}_{\mathrm{f}}^{*} ; \mathrm{S}_{\mathrm{t}}\right) \mathrm{d} \mathrm{S}_{\mathrm{t}} \\
& =\left(1-\frac{\mathrm{T}_{\mathrm{j}}}{\mathrm{T}_{0}}\right) \mathrm{M}_{\mathrm{a}}^{2} \frac{\pi \mathrm{S}_{\mathrm{t}}^{2}}{\mathrm{r}^{*}} \mathrm{~d} \mathrm{~S}_{\mathrm{t}} \int_{\mathrm{V}} \mathrm{S}_{\rho, \mathrm{p}^{\prime}}^{*}\left(\mathbf{X}_{\mathrm{f}}^{*}, \mathbf{X}_{\mathrm{s}}^{*} ; \mathrm{S}_{\mathrm{t}}\right) \mathrm{d}^{3} \mathbf{X}_{\mathrm{s}}^{*}, \\
& \mathrm{~S}_{\rho, \mathrm{p}^{\prime}}^{*}=\frac{\mathrm{S}_{\rho, \mathrm{p}^{\prime}}}{\left(\rho_{0} a_{0}\right)^{2}}
\end{aligned}
$$

In the present experimental program the principle idea of correlating flow fluctuations to the sound pressure fluctuations has been extended to include some other variables, which do not necessarily abide by the framework of Lighthill's equation. For example, efforts are made to correlate $\rho v v$ fluctuations not only with microphone signal from $90^{\circ}$ to jet axis but to $30^{\circ}$ position as well. Inquires are made as how do various other quantities, such as various Reynolds decomposed terms of $\rho u u: \bar{\rho} \bar{u} u^{\prime}, \rho^{\prime} \bar{u} u^{\prime}$ etc. correlate with sound pressure fluctuations. These additional efforts resulted in some interesting results.

\section{Fundamentals of flow measurement using Rayleigh scattering principle:}

A simplistic description of the measurement process, using laser induced Rayleigh scattering is schematically shown in figure 2 . When a laser beam is allowed to pass through a gas, the molecules present in the gas cause inelastic and elastic light scattering. The inelastic part is called Raman scattering and the elastic part Rayleigh scattering. The Rayleigh scattering process describes most ( 99\%) of the molecular scattered light. In the present experiment scattered light is collected and spectrally resolved to measure velocity. Since the Doppler shift frequency is relatively small, a narrow linewidth incident laser beam is necessary to resolve Rayleigh spectrum. Even if the gas medium is stationary, the random thermal motion of the gas molecules creates a wide range of Doppler shift - resulting in a spectral broadening of the collected light. The Full Width at Half Maximum (FWHM) depends on the distribution of molecular velocities and, therefore, is a measure of gas temperature. In the case of a moving gas media, the bulk motion is superimposed on the random velocity of the individual molecules; therefore separation between the peaks of the incident laser line and the Rayleigh spectrum provides a measure of the bulk velocity. Finally, the total light under the Rayleigh spectrum is proportional to the molecular number density and provides a measure of gas density. A single Rayleigh spectrum carries information of one component of gas bulk velocity, temperature and density. This basic principle has been used in the past to measure time-averaged quantities (Panda \& Seasholtz 1999, Forkey, Lempert \& Miles 1998, Elliott \& Sammimy 1996). Since density variation modulates the total scattered light, unsteady density fluctuations are easier to measure (Panda \& Seasholtz, 2002 among others). Extension of the Rayleigh scattering technique to measure unsteady velocity fluctuation has remained a challenge. Recently, Seasholtz, Panda \& Elam (2002) have simultaneously measured the time variation of density and velocity fluctuations in free jets. Earlier efforts that culminated towards the present setup can be found in Seasholtz, Panda \& Elam (2001) and Seasholtz $\&$ Panda (1999a, 2000). The present setup is for a pointmeasuring system; a continuous wave laser was used, and scattered light from a point on the beam was collected and analyzed. (It was realized early on that the current limitations of tunable pulsed-lasers and camera systems make an area measurement technique unsuitable for dynamic measurements over a large frequency bandwidth.) The scattered light collected from the probe volume was spectrally resolved by a Fabry-Perot interferometer. To illustrate the approach, first the nature of a fringe formed by the interferometer is shown in figure 3 . The field of view in the image covers a fraction of the free spectral range. At first, a small portion of light directly out of the laser beam is imaged through the interferometer. The narrow line width of the laser makes a sharp, narrow ring in this image (reference image, figure 3a). In contrast, when the Rayleigh scattered light from a moving gas medium is imaged (Rayleigh image, figure $3 b$ ), a different, and diffused ring results. The radial shift in the peak intensity location between the Rayleigh and reference images corresponds to the Doppler shift associated with the bulk motion of the air stream (the diffused nature of the Rayleigh image is due to the thermal broadening). Since the laser frequency, and 
therefore, the reference fringe is fixed, any radial shift of the Rayleigh image indicates an instantaneous change of air velocity. To continuously monitor the ring diameter in the Rayleigh image, Seasholtz et. al. $(2001,2002)$ used an image dissector that split the image into two concentric parts (figure 4), and measured the ratio of light intensity from the inner and the outer parts using two photomultiplier tubes (PMT). A calibration of the intensity ratio from known jet velocities was necessary for later use in unknown flows. To measure density and velocity simultaneously, a total of 3 PMT were used: 2 for measuring velocity as described above and a third one to monitor scattered light power variation which is proportional to the air density fluctuation.

The component of velocity measured using a given optical arrangement depends on the angular position of the collection lenses with respect to the incident laser beam. Figure 5 shows 2 different optical arrangements of the transmission and collection optics for measuring $\mathrm{u}$ and $\mathrm{v}$ velocity components. The Rayleigh scattered light was collected and transmitted to an adjoining room for spectral analysis. Figure 6 shows a schematic of the spectroscopic arrangement which was identical for both velocity components. Since density and a component of velocity was measured simultaneously, either $\rho(t), u(t)$ or $\rho(t), v(t)$ were measured using the two different collection arrangements. Figure 5(b) presents a scattering diagram pertinent to the radial $\mathrm{v}$ component measurement. $\mathbf{k}_{\mathrm{i}}$ is the incident wave vector normal to the jet flow direction, $\mathbf{k}_{\mathrm{s}}$ is the scattered wave vector pointing towards the collection optics and, $\mathbf{k}=\mathbf{k}_{\mathrm{s}}-\mathbf{k}_{\mathrm{i}}$, is the scattering vector. The arrangement measures Doppler shift, $f_{d}=\mathbf{k} . \mathbf{U} / 2 \pi$ from the radial velocity component $\mathrm{v}$. Similar diagram for the optical arrangement of figure 5(c) shows that the measured Doppler shift corresponds to $-\mathrm{u}$ component. The calibration process accounted for the sign reversal.

The image formed by the Fabry-Perot interferometer depends on the optical frequency distribution of the scattered light, properties of the interferometer, called instrument function, diameter of the optical fiber used for transmitting collected light and focal lengths of the collimating and fringe forming lenses of figure 6 . A detailed discussion can be found in Seasholtz, Panda \& Elam (2002). In brief, the optical spectrum of the Rayleigh scattered light $\Re\left(v-v_{0}\right)$ carries information on the velocity distribution of gas molecules. For a low density gas the spectrum is given as:

$$
\begin{aligned}
& \Re\left(v-v_{0}\right) \mathrm{df} \\
& =\frac{2 \sqrt{\pi}}{a_{\mathrm{p}} \mathrm{k}} \exp \left[-\left\{\frac{2 \pi\left(v-v_{0}\right)-\mathbf{k} \cdot \mathbf{U}}{a_{\mathrm{p}} \mathrm{k}}\right\}^{2}\right] \mathrm{df}
\end{aligned}
$$

Where, $v_{0}$ is the incident laser frequency and $\alpha_{p}$, the "most probable molecular speed," is related to the local sound speed $\alpha$ and ratio of specific heat $\gamma$ through $\alpha_{\mathrm{p}}=(2 / \gamma)^{1 / 2} \alpha$. An examination of the Gaussian distribution shows that the width of the spectrum depends on $\alpha_{\mathrm{p}} \mathrm{k}$, which is related to $\sqrt{T}$ and the peak is shifted by the Doppler shift k.U. The image formed, after the Rayleigh light is passed through a Fabry-Perot interferometer, is basically a convolution of the Rayleigh spectrum with the instrument function $\mathrm{I}_{\mathrm{FP}}\left(\nu, \theta_{\mathrm{r}}\right)$. The light power distribution $P_{I}$ at any position $(r, \theta)$ in the image plane is given as:

$$
\mathrm{P}_{\mathrm{I}}(\mathrm{r}, \theta)=\mathrm{P}_{\Re} \iint \Re(v) \mathrm{I}_{\mathrm{FP}}\left(v, \theta_{\mathrm{r}}\right) \mathrm{d} v \mathrm{dA}
$$

Where, $P_{\Re}$ is the total Rayleigh scattered power, and $\theta_{\mathrm{r}}$ is the angle made by a light ray reaching the elementary area $\mathrm{dA}$ in the image plane with optical axis. In the current setup this image, formed at the focal plane of the fringe-forming lens, was dissected into two parts by a concentric, tilted mirror assembly (image dissector in figure 6) and measured by two PMT. The intensity variations from the two parts were measured from known velocity flows. It was found that the intensity variations in either part of the image can be modeled by $2^{\text {nd }}$ order polynomials. If $\mathrm{N}_{2}$ and $\mathrm{N}_{3}$ denote photo-electron count rates from the inner and outer PMT then:

$\mathrm{N}_{2}=A_{\mathrm{i}}+u B_{\mathrm{i}}+u^{2} \mathrm{C}_{i}, \quad \mathrm{~N}_{3}=A_{\mathrm{o}}+u B_{\mathrm{o}}+u^{2} \mathrm{C}_{\mathrm{o}}$

Where, $\mathrm{A}_{\mathrm{i}}, \mathrm{B}_{\mathrm{i}}, \mathrm{C}_{\mathrm{i}}, \mathrm{A}_{\mathrm{o}}, \mathrm{B}_{\mathrm{o}}$ and $\mathrm{C}_{\mathrm{o}}$ are calibration constants. The velocity component $u$ is measured from a ratio of the two counts, $\mathrm{R}=\mathrm{N}_{3} / \mathrm{N}_{2}$ :

$$
\mathrm{u}=\frac{-\left(R B_{i}-B_{o}\right)+\sqrt{\left(R B_{i}-B_{o}\right)^{2}-4\left(R A_{i}-A_{o}\right)\left(R C_{i}-C_{o}\right)}}{2\left(\mathrm{RC}_{\mathrm{i}}-\mathrm{C}_{\mathrm{o}}\right)}
$$

Note that velocity is calculated from a ratio of two counts $\mathrm{R}=\mathrm{N}_{3} / \mathrm{N}_{2}$, which cancels out changes in overall scattering intensity associated with flow density variation. Also the physically meaningful, negative root of the quadratic equation is considered. The above analysis does not account for changes due to temperature fluctuations on Rayleigh spectra. A numerical uncertainty analysis (Seasholtz et. al. 2001), however, demonstrates that such an effect is small compared to the bigger change associated with velocity fluctuations.

Density variations were measured by splitting off about $10 \%$ of the collected light using a beam splitter (BS1 in figure 6) before the rest is passed through the interferometer. The power variation in this split part is measured by PMT1. It is known that for a fixed optical setup and a fixed composition of gases the total scattered light is directly proportional to the gas density: $\mathrm{P}_{\Re} \propto \rho$. If the photo-electron count rate from PMT1 is $\mathrm{N}_{1}$ then:

$$
\mathrm{N}_{1}=\mathrm{C}_{\rho 1}+\rho \mathrm{C}_{\rho 2} \text {, }
$$


Where, $\mathrm{C}_{\rho 1}$ and $\mathrm{C}_{\rho 2}$ are calibration constants determined from measurements in know density flows.

\section{EXPERIMENTAL SET-UP}

Experiments were performed at NASA Glenn Research Center using three different nozzles (one convergent and two convergent-divergent) operated at Mach numbers, $M$ $=0.95,1.4$ and 1.8. The convergent-divergent nozzles were designed by the method of characteristics and their geometries were reported in Panda \& Seasholtz (1999). The operating conditions are described in Table I. All nozzles were $25.4 \mathrm{~mm}$ in exit diameter. The jet facility used a continuous supply of unheated compressed air. The facility was located in a large test chamber, which was not anechoic per se, but acoustic absorbent material was placed around the vicinity of the nozzle and in the ceiling and walls of the test cell to minimize reflection. Two 1/4-inch microphones were used to measure sound pressure fluctuation spectra. The microphones were mounted on an arc that allowed positioning at a distance of 50 diameters from nozzle lip and polar angles from $30^{\circ}$ to $90^{\circ}$ to the jet axis with $10^{\circ}$ increments. For the bulk of the experiment one of the microphones was kept fixed at $30^{\circ}$ to the jet flow direction and the other at $90^{\circ}$. The Rayleigh scattering system is somewhat elaborate and the following provides a description.

An in-depth discussion of the Rayleigh set-up can be found in Seasholtz, Panda \& Elam (2001, 2002). Following is a brief discussion of some of the central features. The optical system was built in two parts. The first one is around the jet facility for transmitting laser light and collecting the scattered light (figure 5). The scattered light was then passed through $0.55 \mathrm{~mm}$ diameter optical fiber to a quiet room where the second part, consisting of spectroscopic and photon counting electronics, were placed (figure 6). The splitting of the setup is a part of special attention that had to be taken for successful implementation of the optical setup. Care had to be taken to reduce dust particles in the air streams, to minimize the effect of vibration on the optical equipment and to stabilize the interferometer from temperature and vibration induced drifts. To reduce dust particles the dry air, supplied to the facility from a central high-pressure facility, was passed through sub-micron filters. This made the primary jet air very clean. To clean the entrained ambient air, an additional air blower \& filter system was installed that provided a large, $200 \mathrm{~mm}$ diameter, low-speed $(\sim 20 \mathrm{~m} / \mathrm{s})$ co-flow around $25.4 \mathrm{~mm}$ primary jets.

There were two different optical systems built around the jet facility to measure $\mathrm{u}$ and $\mathrm{v}$ components. Both of these were built over an X-Y traversing unit that carried laser head, transmission and collection optics. The probe volume was moved from point to point in the plume, and thereby, allowing survey over a cross- sectional plane. To maximize utilization of the available light the laser head of the solid-state, frequency-doubled, $\mathrm{Nd}: \mathrm{VO}_{4}$ laser was placed at the bottom part of the set-up (figure 5a). About 5 watts of single mode, $532 \mathrm{~nm}$ wavelength laser light was transmitted through a hollow side beam that contained a half-wave plate, focusing lens, mirrors and baffles. Since Rayleigh scattered light is polarization dependent, the half-wave plate was rotated to align the peak scattering plane with receiving optics. The background scattered light was significantly attenuated by suitable use of baffles and beam-dump. The laser head was placed in the same traversing unit as the rest of the transmission and collection optics. It was found that the noise from the jet created a tonal excitement of the laser line at around $430 \mathrm{~Hz}$. To reduce this excitation an anechoic box was built around the laser head. This box significantly reduced the laser unsteadiness, but a trace remained and manifested in the experimental results. The incident laser paths and positions of the collection optics differed for the two setups to measure radial (figure 5a) and axial (figure 5c, 5d) velocity component. For the former, laser beam was passed normal to the jet axis and the collection optics were placed vertically down to collect scattered light from $90^{\circ}$ to the incident beam as well as jet axis. On the other hand, to measure axial velocity component the laser beam was passed at $45^{\circ}$ to the jet axis, and $90^{\circ}$ scattered light was collected by lenses oriented in the same plane containing the laser beam and the jet axis. The lens combinations for the collection optics were the same for both setups. The scattered light was first collimated by a $300 \mathrm{~mm}$ focal length, $80 \mathrm{~mm}$ diameter achromat, and then focused by a $160 \mathrm{~mm}$ focal length achromat on the face of a $.55 \mathrm{~mm}$ diameter multimode fiber. The combination of the fiber diameter and the magnification ratio of the collection optics fixed the probe volume length to $1.03 \mathrm{~mm}$. Not shown in figure 5 is an additional part of the set-up where a small part of light from the transmitting beam was split off for the purpose of monitoring laser frequency as well as to maintain a close-alignment in Fabry-Perot interferometer. Whenever necessary, a pneumatically actuated mirror was placed in the transmission laser path to divert light towards a diffuser. The diffuser was simultaneously brought in front of the collection fiber and reference light, instead of the Rayleigh scattered light, was passed through the collection fiber.

To protect the spectroscopic equipment and the split mirror assembly from high noise level generated by supersonic jets, they were separately kept in an adjoining quiet room. This second part of the setup is schematically shown in figure 6. Light arriving via optical fiber was collimated by $100 \mathrm{~mm}$ focal length lens L1, and about $10 \%$ was split by BS1 and measured by PMT1. Output from PMT1 provided information on the modulation of total scattered light intensity and therefore, was 
proportional to the air density fluctuations. The rest of the collimated beam was passed through a $70 \mathrm{~mm}$ aperture Fabry-Perot interferometer for spectral analysis. Single wavelength light from an extended source is imaged as consecutive rings (fringes) at the output of the interferometer. However, restriction of field of view, imposed by the fiber diameter, created only one fringe as shown earlier in figure 3 . The fringe-forming lens, which ultimately images the fiber face on the image dissector, was made of two camera lenses with suitable separation for an effective focal length of $2909 \mathrm{~mm}$. The large magnification ratio of the setup created a $16 \mathrm{~mm}$ diameter image of the fiber on image dissector. The image dissector was made of two concentric, and slightly tilted mirrors. The inner one had a diameter of $10 \mathrm{~mm}$ and directed the inner part of the fringe to PMT2, while the $25 \mathrm{~mm}$ diameter outer one directed the outer part of the fringe to PMT3. The ratio of light intensities from PMT2 and PMT3 provided a measure of either axial or radial velocity as described earlier.

The success of the velocity measurement system was critically dependent on stable operation of the interferometer. Slight thermal drift or change in the incident laser frequency displaced the reference fringe (figure 3a) and manifested as an artificial bias in velocity measurement. This made the auto-alignment setup (figure 6) a necessary part. The alignment system was basically a feedback control that first measured the reference fringe diameter and compared it with a prescribed targeted diameter. This function was accomplished by splitting parts of the transmitted light using a 3-prism assembly, and imaging them on a CCD camera. Subsequently, the difference between the targeted to the measured fringe diameter was translated into a change of high voltage supply to the piezo-electric actuators that adjusted interferometer's plate separation. Before every Rayleigh measurement, reference light was collected, passed through the same fiber and optical system, and the autoalignment system was engaged. When the desired fringe diameter was obtained within a tolerance, the reference light collection system around the jet facility and the prism assembly were disengaged, and velocity and density measurement via analysis of Rayleigh scattered light began.

Photoelectron counting electronics were used with all 3 PMT signals. The counting was performed over contiguous bins of prescribed time-duration. The digitization of the microphone signals was synchronized and finally 3 channels of photoelectron count and 1 channel of microphone signals were simultaneously acquired in a Personal Computer. Photo-electron counting was started at the beginning of a clock pulse that also digitized the analog microphone signal. The counting ended at the next clock pulse. Long time records of up to 5 million data points from each PMT and the microphone channel were acquired with a typical sampling rate of $90,000 / \mathrm{sec}$. The data points were converted to physical parameters: instantaneous density, velocity and sound pressure through the usage of proper calibration constants. The Welch method of modified Periodograms (1967) was used to calculate individual spectrum and crossspectral density. Each long record was divided into small, $50 \%$ overlapped, segments; modified periodograms of each segment provided local estimates; average of all local estimates provided the final cross-spectral density. The correlation calculations were also performed via Fourier transform, where individual segments of velocity and sound pressure time histories were Fourier transformed, multiplied and inverse transformed. Average of local estimates produced the final correlation.

\section{RESULTS:}

Calibration and validation: As described before, calibration constants are required to convert photoelectron counts from the 3 PMTs to density and velocity values. For this purpose measurements had to be made in known velocity and density flows. The axial velocity and density in the potential core of jet plumes from a convergent nozzle operated in the subsonic Mach numbers, and CD nozzles at the correct operating conditions, are known from isentropic relations:

$$
\begin{aligned}
& \rho=\frac{\mathrm{P}_{0}}{\mathrm{R}_{0}}\left(\frac{\mathrm{P}_{0}}{\mathrm{P}_{\mathrm{a}}}\right)^{-\frac{1}{\gamma}} ; \mathrm{u}=\mathrm{M} a \\
& \text { where, } \mathrm{M}=\left[\left(\left(\frac{\mathrm{P}_{0}}{\mathrm{P}_{\mathrm{a}}}\right)^{\frac{\gamma-1}{\gamma}}-1\right) \frac{2}{\gamma-1}\right]^{0.5} \\
& \text { and } a=\sqrt{\gamma \mathrm{RT}_{0}}\left(\frac{\mathrm{P}_{0}}{\mathrm{P}_{\mathrm{a}}}\right)^{\frac{1-\gamma}{2 \gamma}}
\end{aligned}
$$

The ambient pressure $\mathrm{P}_{a}$, plenum pressure $\mathrm{P}_{0}$ and Plenum temperature $\mathrm{T}_{0}$ were monitored using pressuretransducers and a thermocouple; for unheated air $\gamma=1.4$. Since the jets exhausted into ambient, the plenum pressure was changed to vary plume Mach number. Axial velocity calibrations were performed in the same plumes where later measurements were performed. For the calibration of the radial component of velocity, however, a separate small nozzle was mounted close to the probe volume. This calibration jet was rotated to align the plume along the radial velocity direction of the primary jet. Figure 7 shows a set of typical calibration curves for density and axial velocity measurements. Parts (a) \& (b) show variation of count rate from the two PMT that measure light intensities from the split images formed after the Interferometer (PMT2 and PMT3 in figure6). 
The solid lines are least square fit of a $2^{\text {nd }}$ order polynomial as described in equation 17 . Figure 7(c) shows ratios of the two counts, measured at different flow velocities along with a solid line representing calculated velocities (equation 18). This plot shows a reasonably good fit to the experimental data. Finally, density calibration is shown in figure 7(d). One noticeable feature is a relatively high scatter of the calibration data from the supersonic Mach 1.4 and 1.8 plumes. Prior density measurements and schlieren photographs in these plumes (Panda \& Seasholtz, 2002) showed the presence of weak shock structures even at the design operating condition. Perhaps weak shocks are impossible to avoid in any free supersonic shear flows. To average out the density and velocity variations across the periodic shock system, data was obtained from various axial stations within the potential core. The data scatter of figure 7 is a reflection of point to point variation in the supersonic plume.

Naturally, this data scatter has added some uncertainty in determining turbulent properties. There were additional minor sources; however, the combined effect of all such sources was masked by the uncertainty from electronic shot noise, inevitable in any optical intensity measurement. This is illustrated in figure 8 where velocity fluctuations spectra measured using the present Rayleigh arrangement is compared with that obtained using a hot-wire probe. This figure shows that the shapes of velocity spectra are similar while the absolute energy level in the spectrum measured by the Rayleigh technique is two and a half times of that measured by hot-wire. The Rayleigh spectra float on constant white noise floors, which are expected consequences of electronic shot noise. The propagation of electronic shot noise in the velocity spectrum is somewhat complicated by the ratio of photoelectron counts, $\mathrm{R}=\mathrm{N}_{3} / \mathrm{N}_{2}$, needed to determine instantaneous velocity. The shot noise randomly changes the ratio and manifests as a noise floor in the velocity spectrum. This constant floor is all that exists in data taken from still ambient air. It should be mentioned that the primary interest of the present work is in cross-correlation between sound pressure fluctuations and turbulent flow fluctuations. Since the noise sources in the two measurements are different, the cross-correlation process eliminates the influence of electronic shot noise and the absolute cross-correlation values are expected to have much smaller level of uncertainty.

Nevertheless, a reasonable estimate of the standard deviation (root-mean-square) of various fluctuating quantities is required to non-dimensionalize the absolute correlation values. For example, velocity fluctuations to far field sound pressure fluctuations are expressed as: $\frac{\left\langle\mathrm{u} ; \mathrm{p}^{\prime}\right\rangle}{\mathrm{u}_{\mathrm{rms}} \mathrm{p}_{\mathrm{rms}}}$, which requires an estimate of $\mathrm{u}_{\mathrm{rms}}$. Such estimates were calculated from spectral data by subtracting the constant shot noise floor shown in figure 7. The noise floor was estimated as the spectral density value at the highest resolved frequency of $50 \mathrm{KHz}$, $\left(\mathrm{P}_{\mathrm{u}}\right)_{\mathrm{f}=50000}$, where it is known that the energy from turbulence fluctuations is small.

$$
\mathrm{u}_{\mathrm{rms}}=\sqrt{\Delta \mathrm{f} \sum\left(\mathrm{P}_{\mathrm{u}}-\left(\mathrm{P}_{\mathrm{u}}\right)_{\mathrm{f}=50000}\right)}
$$

Here, $\mathrm{P}_{\mathrm{u}}$ represents power spectral density of $\mathrm{u}$ fluctuations measured in $(\mathrm{m} / \mathrm{s})^{2} / \mathrm{Hz}$ and $\Delta \mathrm{f}=50,000$ is the frequency range. The rms values measured by this shot noise subtraction process are found to be reasonable. For example, in figure $7(\mathrm{a})\left(\mathrm{u}_{\mathrm{rms}} / \mathrm{U}_{\mathrm{j}}\right)_{\text {hot-wire }}=0.166$ and $\left(\mathrm{u}_{\mathrm{rms}} / \mathrm{U}_{\mathrm{j}}\right)_{\text {Rayleigh corrected }}=0.154$ and in figure $7(\mathrm{~b})$ $\left(\mathrm{u}_{\mathrm{rms}} / \mathrm{U}_{\mathrm{j}}\right)_{\text {hot-wire }}=0.14$ and $\left(\mathrm{u}_{\mathrm{rms}} / \mathrm{U}_{\mathrm{j}}\right)_{\text {Rayleigh corrected }}=0.132$. This subtraction method was used uniformly to estimate $\rho_{\mathrm{rms}}, \mathrm{u}_{\mathrm{rms}}$ and $(\rho u u)_{\mathrm{rms}}$ from respective spectra.

Density and velocity fluctuations spectra: Figure 9 show typical velocity and density spectra obtained from the strongest noise-generating regions of Mach 1.4 plume. All velocity data is non-dimensionalized by the centerline axial velocity $U_{j}$ while density is non-dimensionalized by the ambient value. The puu spectrum of figure 9(d) was obtained by first multiplying instantaneous density and velocity: $\rho u u(t)=\rho(t) u(t) u(t)$ and then taking Fourier transforms of the resulting time series data (note that mean values $\rho$ and $u$ were not subtracted). The density velocity cross-spectrum was obtained by multiplying Fourier transform of individual time traces:

$$
\begin{aligned}
& \mathrm{S}_{\mathrm{u} \rho}(\mathrm{f})=\mathfrak{I}(\rho(\mathrm{t})) * \mathfrak{J}^{*}(\mathrm{u}(\mathrm{t}))=\operatorname{Re}\left\{\mathrm{S}_{\mathrm{u} \rho}\right\}-\mathrm{i} \operatorname{Im}\left\{\mathrm{S}_{\mathrm{u} \rho}\right\} \\
& \text { magnitude }\left|\mathrm{S}_{\mathrm{u} \rho}\right|=\sqrt{\left(\operatorname{Re}\left\{\mathrm{S}_{\mathrm{u} \rho}\right\}\right)^{2}+\left(\operatorname{Im}\left\{\mathrm{S}_{\mathrm{u} \rho}\right\}\right)^{2}}, \\
& \quad \text { phase } \theta_{\text {up }}=\frac{\operatorname{Im}\left\{\mathrm{S}_{\mathrm{u} \rho}\right\}}{\operatorname{Re}\left\{\mathrm{S}_{\mathrm{u} \rho}\right\}}
\end{aligned}
$$

In this equation complex conjugate is represented by the superscript *. The magnitude of the cross-spectral density is plotted in figure 9(e), and phase in 9(f). Note that the sharp spike at very low Strouhal frequency $(430 \mathrm{~Hz}$ in absolute frequency) is due to a spurious oscillation in the laser frequency and should be ignored for further interpretation. It is possible to make multiple interesting observations from figure 9 . First, density fluctuation spectrum is similar to that of axial velocity, while radial velocity shows some difference: $u$ and $\rho$ spectra show a continuously decaying shape, while v-spectrum has a hump shape. This indicates a difference in eddies that produce most energetic fluctuations in axial and radial velocity components. However, data obtained from the shear layer show similarity in shapes for all 3 spectra: $\rho$, $\mathrm{u} \& \mathrm{v}$. That the density fluctuation spectra follows that of axial velocity is somewhat expected, as $u$ fluctuations are more energetic than $v$ fluctuations. The difference 
between $\mathrm{u}$ and $\mathrm{v}$ spectra, however, is unexpected. In the normal mode representation of turbulent fluctuations, which is commonly employed in hydrodynamic stability calculations, it is customary to assume identical spectral distribution for all flow and thermodynamic variables. This obviously is incorrect, at least around the centerline region of the jet, which the present experiment finds to be the strongest noise source. A second observation from figure 9 is that the puu spectrum, as expected, has the same shape as that of $u$ spectrum although in absolute value $(\rho u u)_{r m s}>>u_{r m s}$. Third, the velocity-density crossspectrum has a continuously decaying shape, indicating $\mathrm{u}$ and $\rho$ fluctuations are the best correlated at the larger, lower frequency eddies and progressively de-correlate as the eddy size becomes smaller. Fourth, the flat phase relation of figure 9(f) implies that density and axial velocity fluctuations are in phase for all eddy sizes. Following is a discussion of measurement uncertainties.

As already mentioned, the fundamental source of uncertainty in optical measurement is due to electronic shot noise. Since density spectra are calculated directly from the Fourier transform of photoelectron counts, the effect of electronic shot noise is determinable. A detailed discussion can be found in Panda \& Seasholtz (2002). In essence, like velocity spectra, shot noise adds a constant floor and randomness in density spectra. The latter is significantly reduced in the present experiment by averaging over many segments obtained from 5 million point long data string. The constant noise floor particularly masks the lower energy, high-frequency side of the spectrum. Panda and Seasholtz (2002) used a 2PMT-correlation technique to alleviate this problem. In the present work no such correlation technique is applied. It is estimated that as much as $50 \%$ of spectral energy at $\mathrm{St}=0.2$ may be due to this electronic noise source.

Aside from the shot noise, there are three other smaller sources of noise contributing to velocity spectra. First, a slow random variation in the laser frequency over $30 \mathrm{MHz}\left(.001 \mathrm{~cm}^{-1}\right)$ that translates into $\pm 8 \mathrm{~m} / \mathrm{s}$ velocity fluctuation. Second, the intense sound level produced by the jet caused a periodic variation of the laser frequency at about $430 \mathrm{~Hz}$. As described earlier the laser head was enclosed in an anechoic box to alleviate this problem. Nevertheless, the periodic variation could not be completely eliminated; as evident from $\mathrm{u}, \mathrm{v}$, puu spectra. Finally, the Fabry-Perot stabilization setup, needed to lock the reference fringe at a fixed radial position, was effective only within an error margin. A change in the reference fringe position, from the value used for calibration, translates into a spurious mean velocity in the analyzed signal. Similarly, a random positioning error in the reference fringe results in added energy in the spectral data. A direct estimate of the uncertainty from all such sources is difficult to obtain. Nevertheless, the success of the causality method is critically hinged on noise cancellation obtained in cross-correlating two signals of independent noise sources. This cross-correlation is described in the next section.

\section{Correlation between flow fluctuations and sound} pressure fluctuations: The presentation of correlation data starts with figure 10 , where normalized crosscorrelation plots for $30^{\circ}$ and $90^{\circ}$ microphone angles are presented. The cross-correlation values were calculated via Fourier transform, that is, the cross-spectrum was calculated first, and then an inverse transform was taken to return to the time domain. Neither the flow data, nor the microphone data was shifted in time. The crosscorrelation data show sharp rise at a time delay $\tau_{0}$, which is found to be the time needed for sound waves to travel from the laser probe location to the microphone location, $\tau_{0}=\mathbf{r} / \alpha_{0}$. This confirms that the "cause" $(\rho, \mathrm{v}, \rho \mathrm{vv}$ fluctuations in the jet) produces the "effect" (microphone pressure fluctuations) at a predictable time lag, and in turn provides confidence on the measured data. Figure 10 presents data from 2 different Rayleigh setups: parts (a), (b) \& (c) are from u-setup, parts (d) \& (e) from v-setup. In either case the laser probe was located at the end of the potential core, which was found to be the strongest sound source. There are multiple interesting observations that can be made from figure 10. First, sound pressure fluctuations $\mathrm{p}^{\prime}$ correlates far better with $\mathrm{u}$ or puu fluctuations than v or $\rho v v$ fluctuations. Second, air density fluctuations inside the jet show as good a correlation as puu. Third, sound pressure fluctuations at $90^{\circ}$ to the jet axis correlate very poorly with any flow variables. The $<\rho v v ; p>$ correlation of figure $10(\mathrm{e})$ is of particular interest. The second derivative of $\rho v \mathrm{v}$ represents a longitudinal quadrupole with peak radiation direction $90^{\circ}$ to the jet axis. The radiation from such quadrupoles is unaffected by convective amplification. Therefore, it had been expected that $<\rho v \mathrm{v} ; \mathrm{p}^{\prime}>$ correlation from $90^{\circ}$ microphone would be significant. This obviously was found to be incorrect. Since, $v$ and $\rho v v$ fluctuations are found to be poorly correlated with farfield noise, such data are not presented in the rest of the paper. Fourth, the time duration $\Delta \tau$ over which correlation changes from zero to negative to positive and back to zero is significantly long (Figure 10c). The trailing edge of the correlation is somewhat difficult to determine due to the spurious ripples arising from the reflected waves (discussed in the next paragraph); nevertheless for the particular jet, $\Delta \tau$ is estimated as $0.53 \mathrm{milli}-\mathrm{sec}$. This provides a measure of coherence time of the turbulent eddies responsible for sound radiation. Invoking Taylor's hypothesis, the corresponding coherence length scale is $1_{\text {coher }}=\Delta \tau U_{c}$, where $U_{c}$ is the convective speed. Assuming $\mathrm{U}_{\mathrm{c}}=0.7 \mathrm{U}_{\mathrm{j}}$ the coherence length $1_{\text {coher }} \approx 6 \mathrm{D}$. In other words, the longest eddy 
contributing towards correlation is, on an average, 6 jet diameters long. Therefore, the noise source responsible for sound radiation, at the shallow $30^{\circ}$ angle, is indeed "non-compact." Following is a discussion of uncertainty in correlation data.

As mentioned earlier, the cross-correlation process significantly reduces the shot noise contribution and therefore absolute values of $\left\langle\rho ; p^{\prime}>\right.$ and $\left\langle\rho u u ; p^{\prime}>\right.$ are relatively error free. The primary source of uncertainty is due to the shot noise elimination process used in estimating root-mean-square values: $\rho_{\text {rms }}$, $(\rho u u)_{\text {rms }}$ etc. The shot-noise subtraction process has been described earlier. From repeated measurements and comparison with hot-wire data it is estimated that there is a $\pm 10 \%$ uncertainty in rms calculation, which produces an equal uncertainty in correlation data. A second source of uncertainty is sound reflection from large lenses placed close to the plume. Although most of the optical components and mounts were covered by polyurethane foam to minimize reflection, some could not be covered for obvious reasons. The reflected sound waves arrive at the microphone position at a longer time delay than those reaching directly from the source. The additional ripples after the large primary spike, seen in figure 10, are due to the delayed reflected waves. For the most cases, such as in figure 10, it is easy to separate out the spurious reflected part from the desired direct correlation and is expected to have minimum effect on the maximum correlation value.

It is difficult to cross check the correlation values measured in the present experiment with many of the earlier experiments. As mentioned earlier, a large number of similar investigations in the 1970's used intrusive probes, such as hot-wires or microphones. An examination of data from these experiments shows unreasonably large correlation (in very low Mach number jets), which is rightfully assigned to the additional noise created by probe intrusion. One of the exceptions is the work of Schaffer (1979), who used Laser Doppler Velocimetry to measure u-fluctuations in Mach 0.98 jet and cross-correlated with sound fluctuations measured by a microphone. The $<\mathrm{u}^{\prime} ; \mathrm{p}^{\prime}>$ correlation reported by Schaffer is comparable to the similar, Mach 0.95, data obtained in the present experiment. For example,

Schaffer found $\left[\frac{\left\langle u^{\prime} ; p^{\prime}\right\rangle}{u_{r m s} p_{r m s}}\right]_{\max }=0.03$, when the probe was positioned at $\mathrm{x}=10 \mathrm{D}$ and centerline and microphone was located $30^{\circ}$ to the axis. The same correlation number was obtained in the present experiment with identical probe and microphone locations. This close comparison has provided further confidence on the measured data.
Reynolds decomposition of puu and correlation with sound pressure fluctuations: In high Mach number jets the largest term in the Lighthill stress tensor arises from puu fluctuations, and fig 10 confirms that indeed the highest correlation is measured from this quantity. From the modeling perspective it is of interest to break down the full stress term into time averaged and fluctuating parts and to see how the individual components correlate with the far field noise:

$$
\begin{aligned}
& \rho=\bar{\rho}+\rho^{\prime}, \quad u=\bar{u}+u^{\prime} \\
& \left\langle\rho u u ; p^{\prime}\right\rangle=\left\langle 2 \bar{\rho} \bar{u} u^{\prime} ; p^{\prime}\right\rangle+\left\langle\rho^{\prime} \bar{u} \bar{u} ; p^{\prime}\right\rangle+ \\
& \left\langle 2 \bar{u} \rho^{\prime} u^{\prime} ; p^{\prime}\right\rangle+\left\langle\bar{\rho} u^{\prime} u^{\prime} ; p^{\prime}\right\rangle+\left\langle\rho^{\prime} u^{\prime} u^{\prime} ; p^{\prime}\right\rangle
\end{aligned}
$$

The 5 fluctuating terms in puu decomposition were constructed from the measured $\rho(\mathrm{t}), \mathrm{u}(\mathrm{t})$ time traces and individually correlated with the sound pressure fluctuations from the $30^{\circ}$ microphone. Figure 11 presents this data along with correlation from the full puu term. Clearly, the largest contribution is from the "shear noise" term $2 \bar{\rho} \overline{\mathrm{u}} \mathrm{u}^{\prime}$, and the next important term is $\rho^{\prime} \overline{\mathrm{u}} \overline{\mathrm{u}}$ : the other $1^{\text {st }}$ order term. The terms which are $2^{\text {nd }}$ and $3^{\text {rd }}$ order in fluctuations contribute minimally. A closer examination of figure 11 reveals that the phase of the correlation is different between various order terms. The time delay at which the maximum in $\left\langle 2 \bar{\rho} \bar{u} u^{\prime} ; p^{\prime}\right\rangle$ correlation occurs is different from that in $\left\langle 2 \bar{\rho} u^{\prime} u^{\prime} ; p^{\prime}\right\rangle$ correlation. In general, correlation with first and third order terms are nearly in phase while the second order terms are opposite in phase. Therefore, a simple sum of the +ve maxima values in the constituent correlations do not add up to value measured in $<\rho u u ; p^{\prime}>$ correlation. The relative magnitudes and phase relation in the correlation data is further explored in Table II, where numerical values of contribution from various terms at the time delay corresponding to $\left\langle\rho u u ; p^{\prime}\right\rangle_{\max }$ are presented for various Mach number conditions. The observations made in the earlier discussions are once again supported by the numerical values.

From the jet noise modeling perspective, there are three significant observations, which may lead to further improvements of the existing models. The first observation is about the Lilley's equation (1974) which has been used to model jet noise (Khavran, Bridges and Freund 2002, Goldstein 2001 and others). For a transversely sheared flow, i.e, a flow where all mean velocity and temperature gradients, except that along the radial direction $\mathrm{du} / \mathrm{dr}$, is absent, the Lilley's equation can be written as (Goldstein, 1976, Colonius, Lele \& Moin 1997): 


$$
\begin{aligned}
& \frac{\mathrm{D}_{0}}{\mathrm{Dt}}\left(\mathrm{a}_{0}^{2} \nabla^{2} \Pi-\frac{\mathrm{D}_{0}^{2} \Pi}{\mathrm{Dt}^{2}}\right)-2 a_{0}^{2} \frac{\mathrm{du}}{\mathrm{dr}} \frac{\partial^{2} \Pi}{\partial \mathrm{x} \partial \mathrm{r}}= \\
& -\frac{D_{0}}{D t}\left(\frac{\partial^{2} u_{i}^{\prime} u_{j}^{\prime}}{\partial x_{i} \partial x_{j}}\right)+2 \frac{d u}{d r} \frac{\partial^{2} v^{\prime} u_{i}^{\prime}}{\partial x \partial x_{i}}+\text { other smaller terms } \\
& \text { where, } \frac{\mathrm{D}_{0}}{\mathrm{Dt}}=\frac{\partial}{\partial \mathrm{t}}+\mathrm{u}(\mathrm{r}) \frac{\partial}{\partial \mathrm{x}} ; \quad \Pi=\frac{1}{\gamma} \ln \frac{\mathrm{p}}{\mathrm{p}_{0}}
\end{aligned}
$$

In the above equation $\mathrm{u}_{\mathrm{i}}^{\prime}=\mathrm{u}^{\prime}, \mathrm{v}^{\prime}, \mathrm{w}^{\prime}$ are the fluctuating velocity components, $x_{i}=x, r, x_{3}$ is the co-ordinate system, $\mathrm{p}$ is static pressure which is normalized by a suitable reference $\mathrm{p}_{0}$. This is a convected wave equation with the right hand side representing the sound source.

Note that the source terms are second order in fluctuations. Goldstein (2001) provides the significance of this arrangement: "The dominant part of the Lighthill source term is quadratic in the total flow velocity, which can be decomposed into a mean plus a fluctuating component. The source function therefore contains terms that are both linear and quadratic in the fluctuating velocity components. Lilley (1974) argued that the linear terms, which are typically much larger than the quadratic quantities, do not actually radiate any sound and should, therefore, not be included in the source function, since they would tend to dominate over the much smaller quadratic terms which are the true sources of sound." The experimental data of figures 10, 11 and Table II clearly contradicts above conviction. Derivation of equation 24 actually requires moving term linear in fluctuation to the left hand side of the equation and rearranging using the momentum equation. This step perhaps, has mixed the source terms with the propagation terms. There is a caveat to the above discussion: in the following it will be demonstrated that the correlation measured in the present experiment is primarily due to the large coherent structures; contributions from the fine grained turbulence fall below the experimental noise floor. Therefore, the above discussion of limitations strictly applies to the noise component produced from the large scales only.

The second modeling issue is the frequently used assumption of constant flow density in evaluating the density*velocity*velocity product term, i.e., $\rho u u \approx \bar{\rho} u u$. All earlier studies employing the causality technique also used the same assumption. The present data, in contrast, show that the neglected $\rho^{\prime} \overline{\mathrm{u}} \overline{\mathrm{u}}$ term contributes from $10 \%$ to $25 \%$ towards $<$ puu; $p^{\prime}>$ correlation. Therefore, density fluctuations should be included in modeling of the turbulence stresses. The third and the final observation is related to the first and is about modeling the space time correlation $<\operatorname{\rho uu}\left(\mathrm{X}_{\mathrm{s}}, \mathrm{t}^{\prime}\right)$; $\rho \mathrm{uu}\left(\mathrm{X}_{\mathrm{s}}+\xi, \mathrm{t}^{\prime}+\tau\right)>$ required for calculating the far field sound (equation 4 ). Following the intuition mentioned earlier, models depend on the length and time scales calculated from the second order $u^{\prime} u^{\prime}$ fluctuations: $<\mathrm{u}^{\prime} \mathrm{u}^{\prime}\left(\mathrm{X}_{\mathrm{s}}, \mathrm{t}^{\prime}\right) ; \mathrm{u}^{\prime} \mathrm{u}^{\prime}\left(\mathrm{X}_{\mathrm{s}}+\xi, \mathrm{t}^{\prime}+\tau\right)>$ (for example, Khavaran, Krejsa \& Kim, 1992). The present experiment, on the other hand, shows the largest contributions, towards the noise radiated close to the jet axis, are from the first order fluctuations $u^{\prime}$ and $\rho^{\prime}$. Therefore, the required length and time scales should be calculated from $<u^{\prime}\left(X_{s}, t^{\prime}\right) ; u^{\prime}\left(X_{s}+\xi, t^{\prime}+\tau\right)>$ and $<\rho^{\prime}\left(X_{s}, t^{\prime}\right) ; \rho^{\prime}\left(X_{s}+\xi, t^{\prime}+\tau\right)>$ correlations. This is true for predicting noise from large organized structures.

Comparative study of correlation at various microphone angles: Figure 12 shows variations in the maximum positive correlation coefficient, when the microphone polar angle is varied from $30^{\circ}$ to $90^{\circ}$ while the laser probe volume was kept fixed at the indicated $(\mathrm{x}, \mathrm{r})$ location. The angles are measured from the downstream direction. The microphone radial distance from the nozzle was kept fixed at 51 diameters. Interestingly, sound pressure fluctuations from the shallowest polar angle of $30^{\circ}$ show the highest correlation. The correlations drop sharply to the noise floor at $60^{\circ}$ and higher angles. The present correlation study is identifying turbulent fluctuations that radiate close to the jet axis. The absence of correlation at higher polar angles also implies existence of a second mechanism of noise generation which the present point measurement technique is unable to detect. This is discussed at the end of this section.

Comparative study of correlation from various parts of Jets: For this part of the study the microphone was kept fixed, at 50 diameters and $30^{\circ}$ to the jet axis and the laser probe was moved from point to point in the plume. Figure 13 shows the variation in peak correlation when the probe was moved along the jet centerline. The highest correlation values were measured just downstream of the end of the potential core, perhaps due to merging and fragmentation of large organized structures present in the peripheral shear layer. Note that the potential core lengthens with Mach number due to a decrease in shear layer growth rate. The end appears around $\mathrm{x} / \mathrm{D}=6,7$ and 9 for Mach $0.95,1.4$ and 1.8 jets respectively (Panda \& Seasholtz, 2002). The variation of the correlation coefficient along the shear layer $(\mathrm{r} / \mathrm{D}=0.45)$ is very different from that along centerline. For figure 14, the microphone was again kept fixed at the same $30^{\circ}$ location, while the probe volume was moved axially from point to point along $\mathrm{r} / \mathrm{D}=0.45$. These data show a sharp difference between subsonic and supersonic plumes. In the subsonic Mach 0.95 plume all correlations fall below experimental noise floor, while in the supersonic Mach 1.4 and 1.8 cases significant correlation is measured. The difference between the centerline and the shear layer behavior prompted a study of radial dependence of the correlation coefficients. Figure 15 shows that indeed the highest correlation is measured at the centerline. The 
$<$ puu; $p>$ correlation progressively falls to the noise floor for $\mathrm{r} / \mathrm{D}>0.6$. The $<\rho ; \mathrm{p}>$ correlation, however, shows an interesting trend: a low correlation, yet higher than the noise floor, is measured when the probe volume is positioned outside the turbulent plume. This small value remained nearly constant beyond $\mathrm{r} / \mathrm{D}=0.6$. A qualitative explanation is as follows. Sound waves can be thought as density fluctuations. In Mach 1.4 jet, where some turbulent eddies attain supersonic speed relative to the ambient, the shock waves associated with the individual eddies propagate directly to the far field. The presence of this Mach wave emission process, perhaps, results into the measurable correlation between the near field (existing just outside the jet boundary) air density fluctuations and the far field sound pressure fluctuations.

Frequency analysis of cross-correlation data, crossspectral density: So far all correlation data were presented in time domain. Figure 16 presents data in frequency domain. The cross-spectral density values are normalized by time averaged plume and ambient properties: $\alpha_{0}, \mathrm{U}_{\mathrm{j}}$, and $\rho_{0}$, as described in equations 12 and 13 . Note that figures 16(b1) and (b2) were obtained from the same data set used in the spectral plots of figure 9 and correlation plots of figures 10 and 11. The first point to be discussed is that figure 16 provides evidence to the claim that crosscorrelation data are significantly free of the shot-noise effects. Unlike $\rho$ and $\rho u$ spectra in figure 9 , the $S_{\rho, p}$, and $S_{\text {puu,p }}$, cross-spectra rise 2 to 3 decades above the noise floor. Moreover, the sharp peak at $430 \mathrm{~Hz}$ seen in puu spectra is absent in $\mathrm{S}_{\text {puu, }}$, cross-spectra. The second point to be made is that the turbulent fluctuations providing most of the correlations lie in the nominal Strouhal number range $0 \leq \mathrm{St}<1.0$, with the peak value around $\mathrm{St}=0.2$. All of these cross-spectra demonstrate that the measured correlations are mostly due to the large organized structures present in the plume. Contribution from small scale fluctuations fall below the noise floor. Another noticeable trend is the similarity in the overall shapes of $\mathrm{S}_{\rho, \mathrm{p}}$, and $\mathrm{S}_{\rho u u, p}$, cross-spectra over the entire Mach number range.

Contribution to far field noise from $<\rho ; p^{\prime}>$ and $<\rho u$; $p^{\prime}>$ correlation: The causality principle, described in the introduction, can be used to back calculate far field sound pressure fluctuations from the measured cross-

correlations. This obviously is not a modeling approach, yet serves the purpose of estimating the fraction of noise generated from various regions of the jet. Figure 17 presents one such effort to calculate auto-spectrum of sound pressure fluctuations at the $30^{\circ}$ location (and 50D away from the nozzle exit) from a single point crosscorrelation measurement. The laser probe was placed at the centerline and downstream of the potential core where maximum correlation is measured. The probe and microphone locations are the same for which correlation and spectral data are presented earlier. Figures 17(c) and (d) show a comparison of the calculated auto spectrum with the actual measured profile. Recall that the unevenness in the measured auto-spectra is an artifact of reflection from large lenses placed close to the plume. The calculation procedures involve multiplication of cross spectral densities, $S_{\rho, p}$, and $S_{\rho u u, p}$, by various terms, most importantly by frequency squared, as described in equations 13 and 14 . The dashed curve represents left hand sides of the equations and the solid curve the right hand side. Note that the causality equations require an integration of all cross-spectra measured over the entire jet plume. This has not been attempted in the present work. Instead the calculated auto spectrum should be interpreted as effectiveness of a unit volume of flow, centered at the laser probe, in creating sound pressure fluctuations at the microphone location. Interestingly, figure 17(d) shows that the measured correlations are so high that such a unit volume by itself produces more noise than the measured auto-spectrum. Figure 17(c) shows that the dipole contribution from density fluctuations is about $10 \%$ of the total measured sound pressure. Perhaps the only way the extraordinary efficiency of a single volume of turbulent fluctuations to generate noise can be reconciled, is by assuming phase cancellations from different regions of the jet.

\section{Plausible reason for the directional variation of correlation coefficients: It is known that the sound} pressure fluctuations measured by a far field microphone is due to a sum of radiation from a large number of turbulent eddies distributed in the entire jet plume. Therefore, at first it was expected that individual point correlations, $<\rho ; p^{\prime}>$ and $<\rho u u ; p^{\prime}>$, would be very small. The experimental data, however, shows significantly high values at shallow angles and for the supersonic jet velocities. It is conjectured that present experiment picks up contribution from the coherent eddies and is unable to determine contribution from fine scale, small eddies. By definition, coherent eddies have long spatial coherence. The radiation from various spatial locations of such eddies are expected to be phase related (figure 18); therefore, a single point correlation reflects contribution from the entire eddy. Frequency analysis of crosscorrelation data demonstrates that the measured correlations are from low Strouhal frequency fluctuations. Such fluctuations are known to be due to large organized structures which radiates primarily in the downstream direction close to the jet axis (Morris \& Tam, 1979, Tam \& Burton, 1984). Theoretical calculations (Michalke 1977, 1983) show that the higher the spatial correlation of turbulence, the narrower is the radiation angle. The quick drop in correlation with an increase of microphone polar angle, seen in figure 12 , 
further supports that the large spatially coherent structures have mostly contributed towards the measured correlations. Panda and Seasholtz (2002) have presented schlieren photographs of the same jet studied in the present paper. These photos demonstrate the presence of Mach wave emission (due to supersonic convective speed of some eddies) for the supersonic cases. The shock waves attached to the supersonically convected eddies makes an almost one to one connection between the near field turbulence and the far field sound pressure fluctuations. This is the reason for significantly higher correlation in supersonic plumes. The radiation fields of small turbulent eddies, however, is expected to be more omni-directional. In addition, they do not have significant coherence. Therefore, contribution from the small scale eddies towards single point $<\rho ; \mathrm{p}^{\prime}>\&<$ puu; $\mathrm{p}^{\prime}>$ correlations are expected to be extremely small, below experimental noise floor. This perhaps explains inability of the present scheme to identify noise sources for $90^{\circ}$ radiation.

\section{SUMMARY AND CONCLUSIONS:}

A recent advancement in molecular Rayleigh scattering technique, to simultaneously measure velocity and density fluctuations in high-speed flows, has been utilized to identify sound sources in supersonic and high subsonic unheated free jets. The particle-free, non-invasive technique involves passing a narrow $\mathrm{CW}$ laser beam through jet plumes and the collection of molecular scattered light from a point on the beam. A part of the collected light is directly measured to detect fluctuating intensity, which is related to air density fluctuations via calibration constants. The rest of the collected light was passed through a Fabry-Perot interferometer to detect Doppler shift associated with one component of jet velocity. The Fabry-Perot was operated in an imaging mode and the fringe formed at the image plane was split into two parts. The ratio of light intensity was related to velocity using another set of calibration constants.

Two separate arrangements of collection optics were used to measure either $\rho$, u or $\rho, \mathrm{v}$ simultaneously. Time histories measured from various points in the plume were Fourier transformed to obtain spectra. A comparison shows that $\rho$ and $u$ spectra are similar in shape while $v$ spectrum is different, especially in the centerline of the jet. The spectral data suffer from a large bias error due to electronic shot noise; yet the correlation data between the turbulent fluctuations and far field sound pressure fluctuations are relatively error-free.

The noise emitted by the jets was measured by microphones placed in the far field and at polar angles from $30^{\circ}$ to $90^{\circ}$ to the downstream direction. The microphone signals $p^{\prime}(t)$ were correlated separately with $\rho(t), u(t), v(t), \rho u u(t)$ and $\rho v v(t)$ signals measured from various points in the flow. The non-intrusiveness of the laser-based technique avoids the probe-interference effects that have plagued previous attempts of source identification via causality method. Some significant observations from this study are the following:

(a) Out of all flow parameters, the puu fluctuations are found to provide the highest correlation with the far field noise. This is closely followed by density $\rho$ fluctuations. Indeed the $<\rho u u ; p^{\prime}>$ and $<\rho ; p^{\prime}>$ correlations are strikingly similar in all respects: absolute magnitudes of normalized correlations, frequency distribution, and dependence on the microphone and probe volume locations. The $\mathrm{v}$ and $\rho \mathrm{v} v$ fluctuations are found to be poorly correlated with $\mathrm{p}$.

(b) The variation of the correlation coefficients with microphone polar angles, and frequency dependence of the cross-spectral data showed that the large scale organized structures mostly contributed towards the correlation coefficients. Cross-spectral analysis of $\rho u u$ and $\mathrm{p}^{\prime}$ fluctuations shows that the highest cross-spectral density is measured from Strouhal frequency, $\mathrm{St} \sim 0.2$. In general, turbulent fluctuations in the frequency range $0 \leq \mathrm{St}<1$ were correlated with far field noise. This Strouhal frequency range is typical of large organized structures. A study of correlation coefficients with microphone angle show that the polar angle closest to the jet axis $\left(30^{\circ}\right)$ provide the highest correlation; the magnitude falls sharply till $60^{\circ}$, beyond which data were mostly below experimental noise floor. Previous analysis (for example Michalke, 1977) has shown that the larger the spatial coherence of turbulence, the narrower the radiation angle. The long spatial coherence is characteristic of organized vortical motion.

(c) The spatial dependence of the correlation coefficients was studied by moving the laser probe volume from point to point in the flow while keeping the microphone fixed (mostly at $30^{\circ}$ ). The strongest soundproducing region is found to lie along centerline and beyond the end of the potential core. For example, in Mach 1.4 jet the highest correlation was measured from centerline and $x / D=10$, the potential core ended at $x / D \approx$ 7. When the laser probe was moved radially, the $\left\langle\rho v v ; p^{\prime}>\right.$ correlation was found to reduce monotonically and fall below the noise floor beyond the edge of the turbulent flow. Correlation from the lip shear layer is found to be Mach number dependent. Significant correlations were measured all along the lip shear layer of supersonic plumes, while the subsonic plumes did not show any such correlation. It is believed that the difference is due to the inception of the "Mach wave" emission process; where some eddies attain convective velocity higher than ambient. The far-field propagation of the shock waves, attached to the eddies, produce the high correlation in supersonic jets. In general, the higher normalized 
correlation values measured in supersonic flow is also due to this Mach wave emission process.

(d) To help various jet noise modeling efforts, Reynolds decomposition was performed on the full puu term, and the individual fluctuating terms were separately correlated with the far field noise. It was found that the first order fluctuations $\left\langle 2 \bar{\rho} \overline{\mathrm{u}} \mathrm{u}^{\prime} ; \mathrm{p}^{\prime}\right\rangle$ and $\left\langle\rho^{\prime} \overline{\mathrm{u}} \overline{\mathrm{u}} ; \mathrm{p}^{\prime}\right\rangle$ provided the largest contribution towards the full correlation $<\rho u u ; p^{\prime}>$. Terms which are second and third order in fluctuations contribute significantly less. This demonstrated, among other things, the limitation of traditional approximation, $\rho u u \approx \bar{\rho} u u$, even in unheated subsonic jets.

(e) According to the causality principle, second time derivatives of $<\rho u u ; p>$ and $<\rho ; p^{\prime}>$ crosscorrelations are related to the $\left\langle\mathrm{p}^{\prime} ; \mathrm{p}^{\prime}>\right.$ auto-correlation of far field sound pressure fluctuations. To avoid taking derivatives of experimental data such calculations were performed in frequency domain where cross-spectra are multiplied by freqency ${ }^{2}$. The principle dictates that a volume integral of all correlations over the plume leads to the far field sound pressure auto spectrum. In this work, a limited effort to determine effectiveness of a unit volume around a single measurement point to produce far field noise was calculated. It was found that the correlation values from the strongest noise producing regions are so high that a single point correlation is capable of producing more noise than the measured far field spectra. This has lead to a conjecture that phase cancellation from sound waves appearing from different parts of large coherent structures ultimately reduces the radiation effectiveness of a single source point.

\section{References}

Armstrong, R. R., Michalke, A. \& Fuchs, H. V. 1977 Coherent structures in jet turbulence and noise. AIAA J. 15 (7), 1011-1017. Elliott, G. S. \& Samimy, M. 1996 Rayleigh scattering technique for simultaneous measurements of velocity and thermodynamic properties. AIAA J. 34 (11), 2346-2352. Fedorchenko, A. T. 2000 On some fundamental flaws in present aeroacoustic theory. J. Sound Vib. 232, 719-782. Ffowcs Williams, J. E. 1973 Technical evaluation report. AGARD CP 131 on Noise Mechanisms, VII - XIX. Forkey, J. N., Lempert, W. R. \& Miles, R. B. 1998 Accuracy limits for planer measurements of flow field velocity, temperature and pressure using filtered Rayleigh scattering. Experiments in Fluids, 24, 151-162.

Goldstein, M. E. 1976 Aeroacoustics, McGraw-Hill International Book Co., New York.

Hussain, A. K. M. F. \& Zaman, K. B. M. Q. 1981 The 'preferred mode' of axisymmetric jet. J. Fluid Mech., 110, 39-71.
Hurdle, P. M., Meecham, W. C. \& Hodder, K. 1974 Investigation of the aerodynamic noise generation region of a jet engine by means of the simple source fluid dilatation model. J. Acoustical Soc. Of America, 56, 1708-1721. Khavaran, A., Krejsa, E. A. \& Kim, C. M. 1992 Computation of Supersonic Jet Mixing Noise for an Axisymmetric CD Nozzle Using k- $\varepsilon$ Turbulence Model. AIAA paper 92-0500, 30 ${ }^{\text {th }}$ AIAA Aerospace Sciences Meeting, Reno.

Lee, H. K., \& Ribner, H. S. 1972 Direct correlation of noise and flow of a jet. J. Acoustic. Soc. Of America. 52, no. 5 (pt. 1) 1280-1290.

Lighthill, M. J. 1954 On sound generated aerodynamically I. General theory. Proc. Royal Soc., A221 564-587.

Lilley, G. M. 1972 The generation and radiation of supersonic jet noise IV. Theory of turbulence generated noise. USAPL TR-72-53.

Michalke, A. 1977 On the effect of spatial source coherence on the radiation of jet noise, J. Sound \& Vib. $\mathbf{5 5}$ (3), 377-394.

Michalke, A. 1983 Some remarks on source coherence affecting jet noise. J. Sound \& Vib. 87(1), 1-17. Morris, P. J., Boluriaan, S., Lilley, G. M. \& Long, L. N. 2002 Two-point cross correlations of turbulence and noise predictions: analysis and simulation. AIAA paper no. 2002-0071

Morris, P. J. \& Tam, C. K. W. 1979 On the radiation of sound by the instability waves of a compressible axisymmetric jet. In Mechanisms of Sound Generation in Flows (ed. E. A. Muller). Springer.

Panda, J. \& Seasholtz, R. G. 2002a Measurement of correlation between density, velocity and density*velocity $^{2}$ with far field noise in high speed jets. AIAA paper 2002-2485.

Panda, J. \& Seasholtz, R. G. 2002 Experimental Investigation of density fluctuations in high-speed jets and correlation with generated noise. J. Fluid Mech., 450, 97-130.

Panda, J., \& Seasholtz, R. G. 1999 Velocity and temperature measurement in supersonic free jets using spectrally resolved Rayleigh scattering. AIAA paper $99-$ 0296.

Proudman, I. 1952 The generation of noise by isotropic turbulence. Proceedings of the Royal Society, A214 119132.

Rackl, R. 1973 Two causality correlation techniques applied to jet noise, Ph. D. thesis, University of British Columbia. 
Richarz, W. G. 1980 Direct correlation of noise and flow of a jet using Laser Doppler. AIAA J. 18(7), 759-765.

Schaffer, M. 1979 Direct measurements of the correlation between axial in-jet velocity fluctuations and far field noise near the axis of a cold jet. J. Sound \& Vib. 64(1) 73-83.

Seasholtz, R. G., Panda, J. \& Elam, K. A. 2002 Rayleigh

Scattering Diagnostic for Measurement of Velocity and

Density Fluctuation Spectra. $40^{\text {th }}$ AIAA Aerospace

Sciences meeting, AIAA paper no 2002-0827.

Seasholtz, R. G., Panda, J. \& Elam, K. A. 2001 Rayleigh

Scattering Diagnostics for Dynamic Measurement of

Velocity Fluctuations in High Speed Jets. AIAA paper no 2001-0847.

Seasholtz, R.G. \& Panda, J. 2000 Rayleigh scattering diagnostic for simultaneous measurements of dynamic density and velocity," AIAA paper 2000-0642.

Seasholtz, R.G. \& Panda, J. 1999a Rayleigh scattering diagnostic for dynamic measurement of velocity and temperature," AIAA paper 99-0641.
Seiner, J. M. 1974 The distribution of jet source strength intensity by means of a direct correlation technique. Ph.D. dissertation, The Pennsylvania State University, Department of Aerospace Engr.

Siddon, T. E. 1973 Noise source diagnostics using causality correlations, AGARD CP 131, Noise Mechanisms 7-1:7-13.

Seiner, J. M. \& Reethof, G. 1974 On the distribution of source coherency in subsonic jets. AIAA paper 74-4.

Tam, C. K. W. 2001 On the failure of the Acoustics

Analogy Theory to identify the correct noise sources. AIAA paper no 2001-2117.

Tam, C. K. W. \& Burton, D. E. 1984 Sound generated by instability waves of supersonic flows. Part 2.

Axisymmetric Jets. J. Fluid Mech. 138 249-271.

Welch, P. D. 1967 The use of fast Fourier transform for the estimation of power spectra: A method based on time averaging over short, modified periodograms, IEEE

Trans. on Audio and Electroacoustics, AU-15 70-73.

Table I. Nominal Operating conditions:

\begin{tabular}{llll}
\hline Sp. heat ratio, $\gamma=1.4 ;$ Total temp., $\mathrm{T}_{0}=300^{\circ} \mathrm{K} ;$ Ambient density, $\rho_{\mathrm{a}}=1.16 \mathrm{Kg} / \mathrm{m}^{3}$, Ambient sound speed, $\mathrm{a}=347 \mathrm{~m} / \mathrm{s}$ \\
\hline $\begin{array}{l}\text { Nozzle type } \\
\text { Minimum shock operation at } \mathrm{M}=\end{array}$ & $\begin{array}{l}\text { Convergent operated at } \\
\mathrm{M}=0.95\end{array}$ & $\mathrm{M} 1.4 \mathrm{CD}$ & $\mathrm{M} 1.8 \mathrm{CD}$ \\
$\begin{array}{l}\text { Reynolds number } \mathrm{Re}_{\mathrm{D}} \\
\text { Jet Velocity } \mathrm{U}_{\mathrm{j}}(\mathrm{m} / \mathrm{s})\end{array}$ & $0.66 \times 10^{6}$ & $1.16 \times 10^{6}$ & $1.88 \times 10^{6}$ \\
$\begin{array}{l}\text { Estimated eddy convection speed } \mathrm{U}_{\mathrm{c}} \\
(\mathrm{m} / \mathrm{s})=0.6 \mathrm{U}_{\mathrm{j}}-0.89 \mathrm{U}_{\mathrm{j}}\end{array}$ & 316 & 411 & 486 \\
$\begin{array}{l}\text { Jet density } \rho_{\mathrm{j}}\left(\mathrm{kg} / \mathrm{m}^{3}\right) \\
\text { Frequency }(\mathrm{kHz}) \text { for } \mathrm{St}=1\end{array}$ & $190-282$ & $247-366$ & $292-433$ \\
\hline
\end{tabular}

Table II. Correlation coefficients between far field sound pressure fluctuations and various Reynolds decomposed terms of puu.

\begin{tabular}{|c|c|c|c|c|c|c|c|c|c|}
\hline $\begin{array}{l}\text { Mach } \\
\text { no. }\end{array}$ & $\begin{array}{l}\text { Prob } \\
\text { e x/D }\end{array}$ & $\begin{array}{l}\text { Prob } \\
\text { e r/D }\end{array}$ & $\begin{array}{l}\text { Mi } \\
\text { c } \\
\text { ang }\end{array}$ & $\frac{\left\langle\text { puu; } \mathrm{p}^{\prime}\right\rangle}{(\rho \mathrm{uu})_{\mathrm{rms}} \mathrm{p}_{\mathrm{rr}}^{\prime}}$ & $\frac{\left\langle 2 \overline{\rho u} u^{\prime} ; p^{\prime}\right\rangle}{(\rho u u)_{r m s} p_{r m s}^{\prime}}$ & $\frac{\left\langle\rho^{\prime} \overline{\mathrm{uu}} ; \mathrm{p}^{\prime}\right\rangle}{(\rho \mathrm{uu})_{\mathrm{rms}} \mathrm{p}_{\mathrm{rms}}^{\prime}}$ & $\frac{\left\langle 2 \overline{\mathrm{u}} \rho^{\prime} \mathrm{u}^{\prime} ; \mathrm{p}^{\prime}\right\rangle}{(\rho \mathrm{uu})_{\mathrm{rms}} \mathrm{p}_{\mathrm{rms}}^{\prime}}$ & $\frac{\left\langle\bar{\rho} \mathrm{u}^{\prime} \mathrm{u}^{\prime} ; \mathrm{p}^{\prime}\right\rangle}{(\rho \mathrm{uu})_{\mathrm{rms}} \mathrm{p}_{\mathrm{rms}}^{\prime}}$ & $\frac{\left\langle\rho^{\prime} u^{\prime} u^{\prime} ; p^{\prime}\right\rangle}{(\rho u u)_{r m s} p_{r m s}^{\prime}}$ \\
\hline 1.8 & 12 & 0 & $30^{\circ}$ & 0.2207 & 0.1635 & 0.0636 & -0.00214 & -0.0025 & 0.003 \\
\hline 1.8 & 6 & 0.45 & $30^{\circ}$ & 0.0572 & 0.0406 & 0.0201 & -0.0022 & -0.0023 & 0.0011 \\
\hline 1.4 & 10 & 0 & $30^{\circ}$ & 0.1919 & 0.1344 & 0.0624 & -0.0021 & -0.0041 & 0.0014 \\
\hline 0.95 & 10 & 0 & $30^{\circ}$ & 0.0682 & 0.0592 & 0.01 & 0.0001 & -0.0019 & 0.0007 \\
\hline 0.8 & 8 & 0 & $30^{\circ}$ & 0.022 & 0.0198 & 0.0037 & -0.0003 & -0.0012 & 0.0003 \\
\hline
\end{tabular}




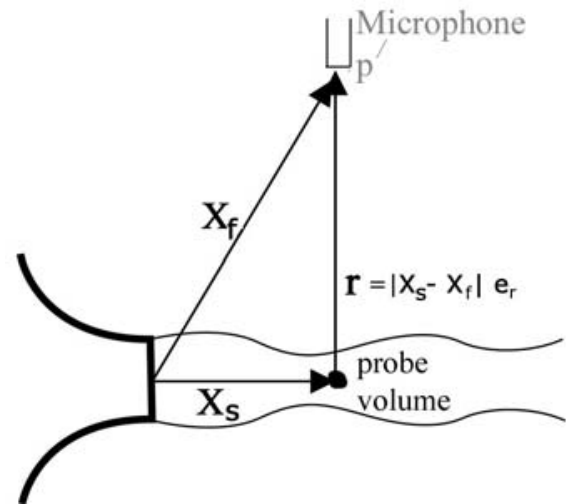

Fig 1. Schematic of coordinate system and microphone locations.

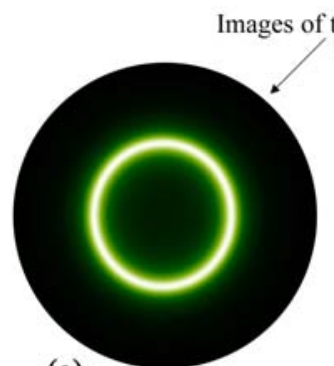

(a)

Fig 3. Fringes formed after passing through FabryPerot Interferometer by (a) incident laser light (b) Rayleigh scattered light.

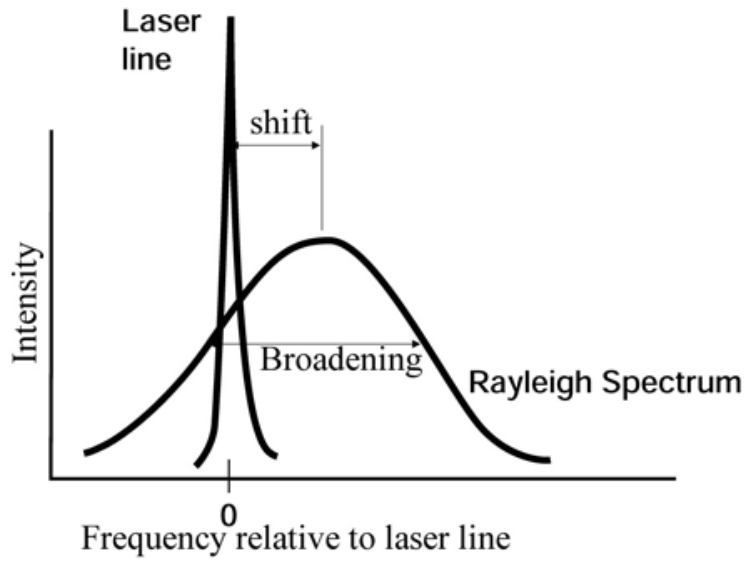

Fig 2. Principle of Rayleigh scattering technique.

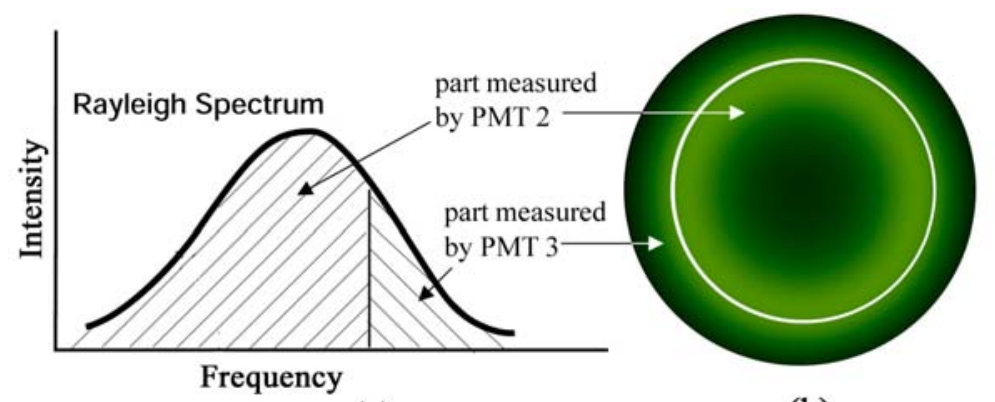

(a)

Fig 4. Principle of instantaneous velocity measurement by Rayleigh scattering technique.

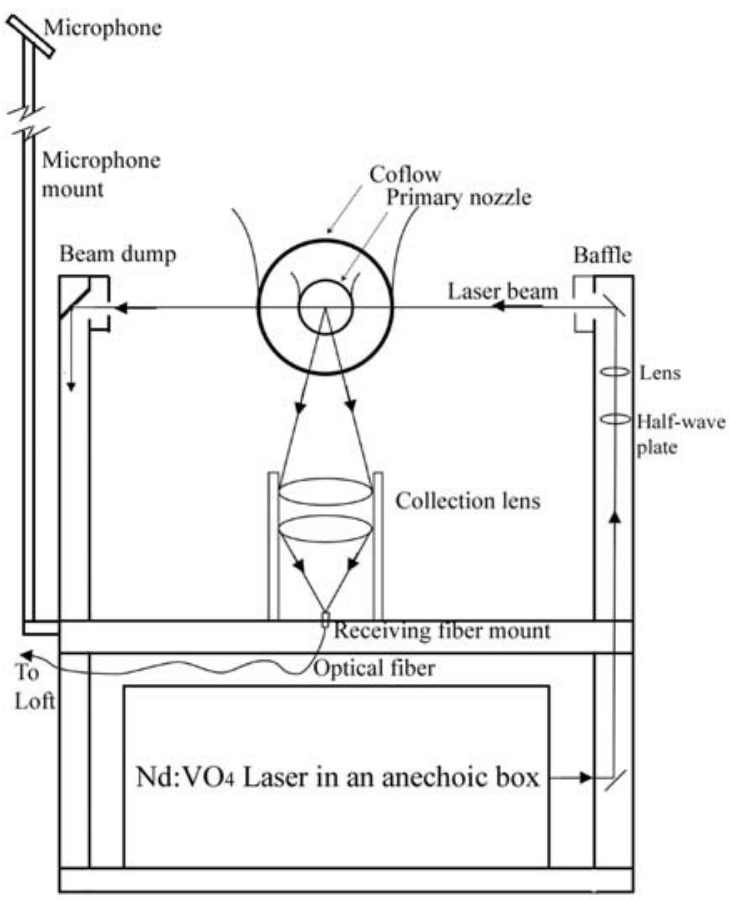

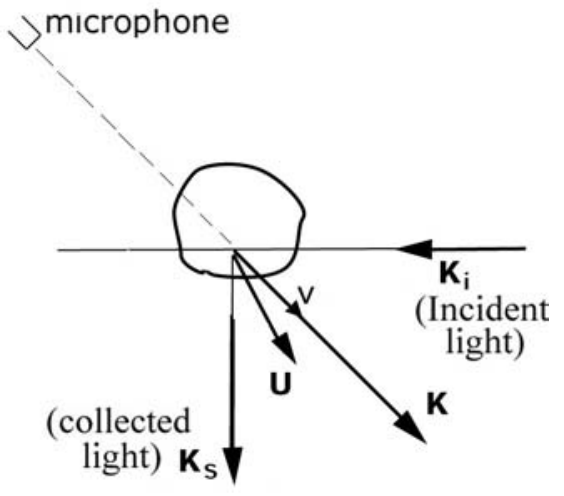

(b)

(a)

Fig 5. (a) Schematic of optical arrangement around jet facility to measure radial v component of velocity. (b) Scattering diagram for $v$ measurement. (c) Top view of optical arrangement to measure axial u component. (d) Side view for $u$ component measurement. 


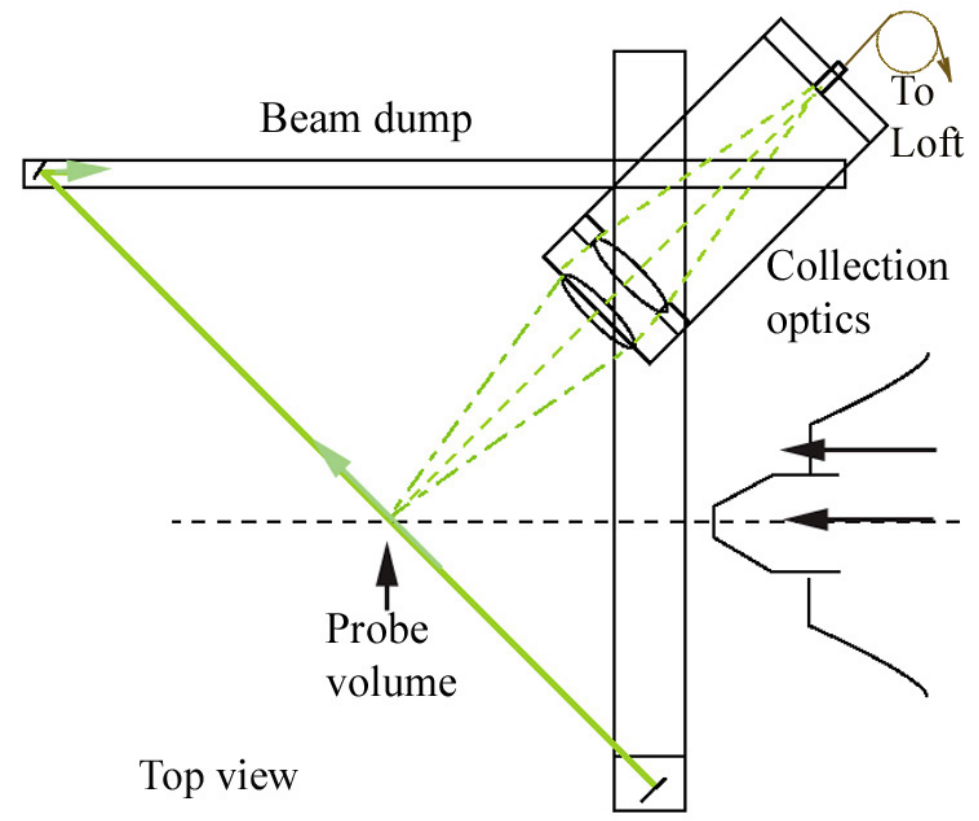

(c)

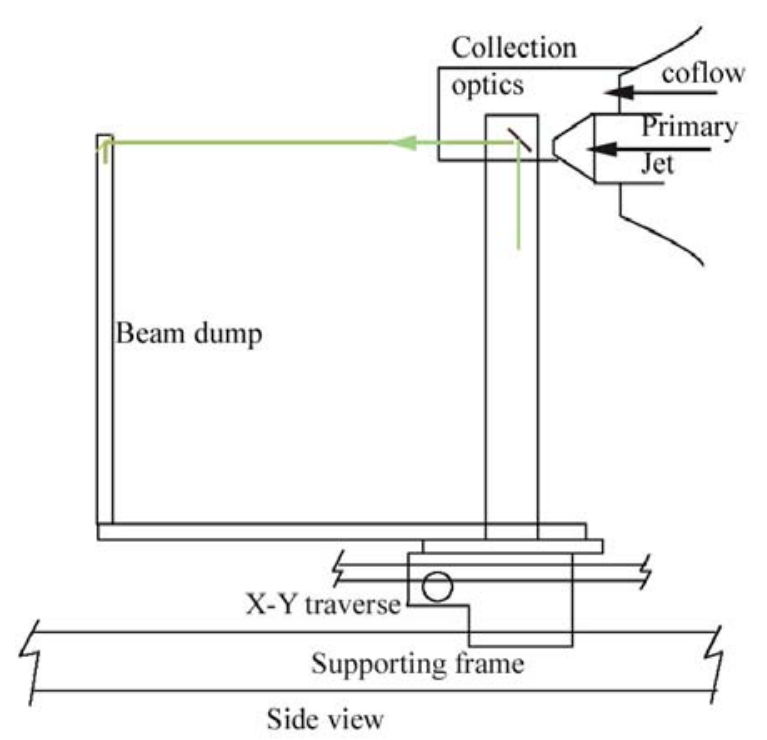

(d)

Fig 5. (continued) (a) Schematic of optical arrangement around jet facility to measure radial v component of velocity. (b) Scattering diagram for v measurement. (c) Top view of optical arrangement to measure axial u component. (d) Side view for u component measurement.

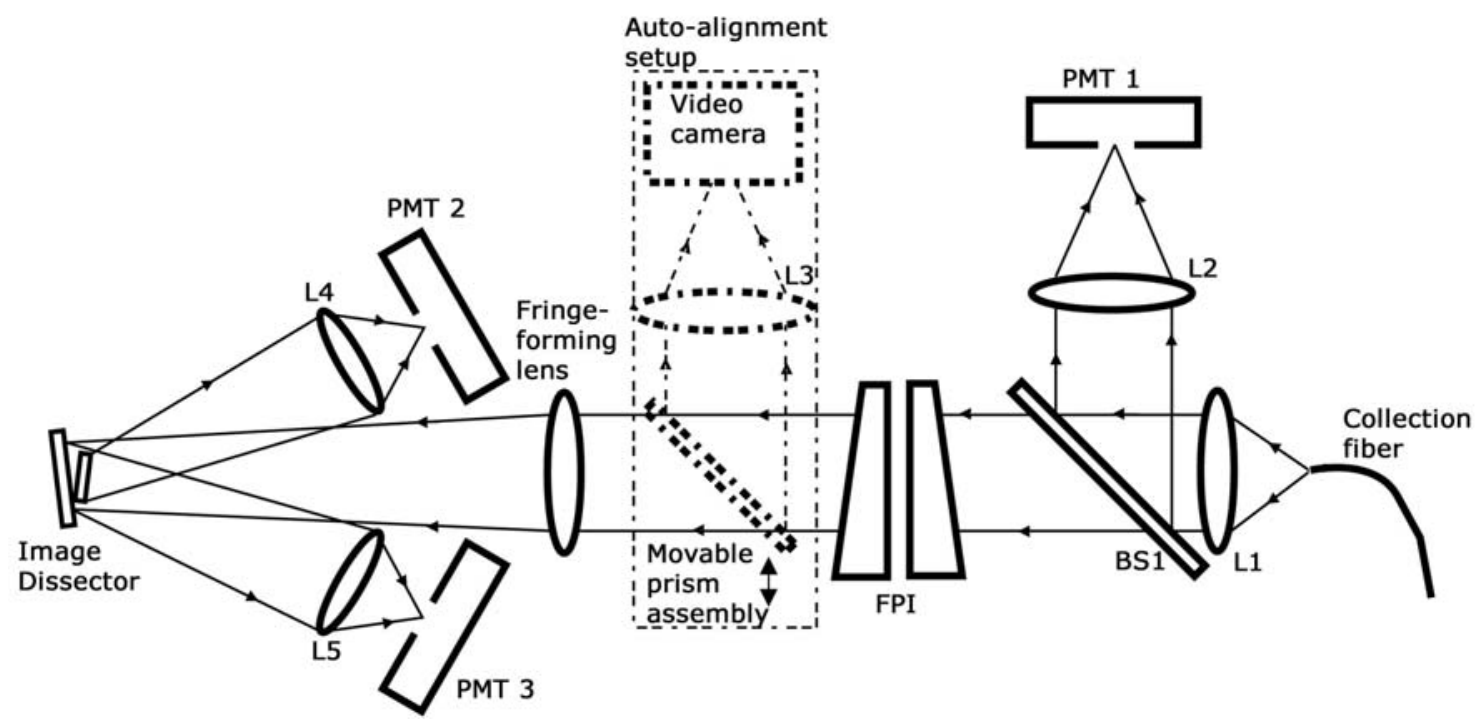

Fig 6. Schematic of optical setup to analyze collected light. L1 to L5 are lenses; BS1 is beam-splitter and FPI is the Fabry-Perot interferometer. 

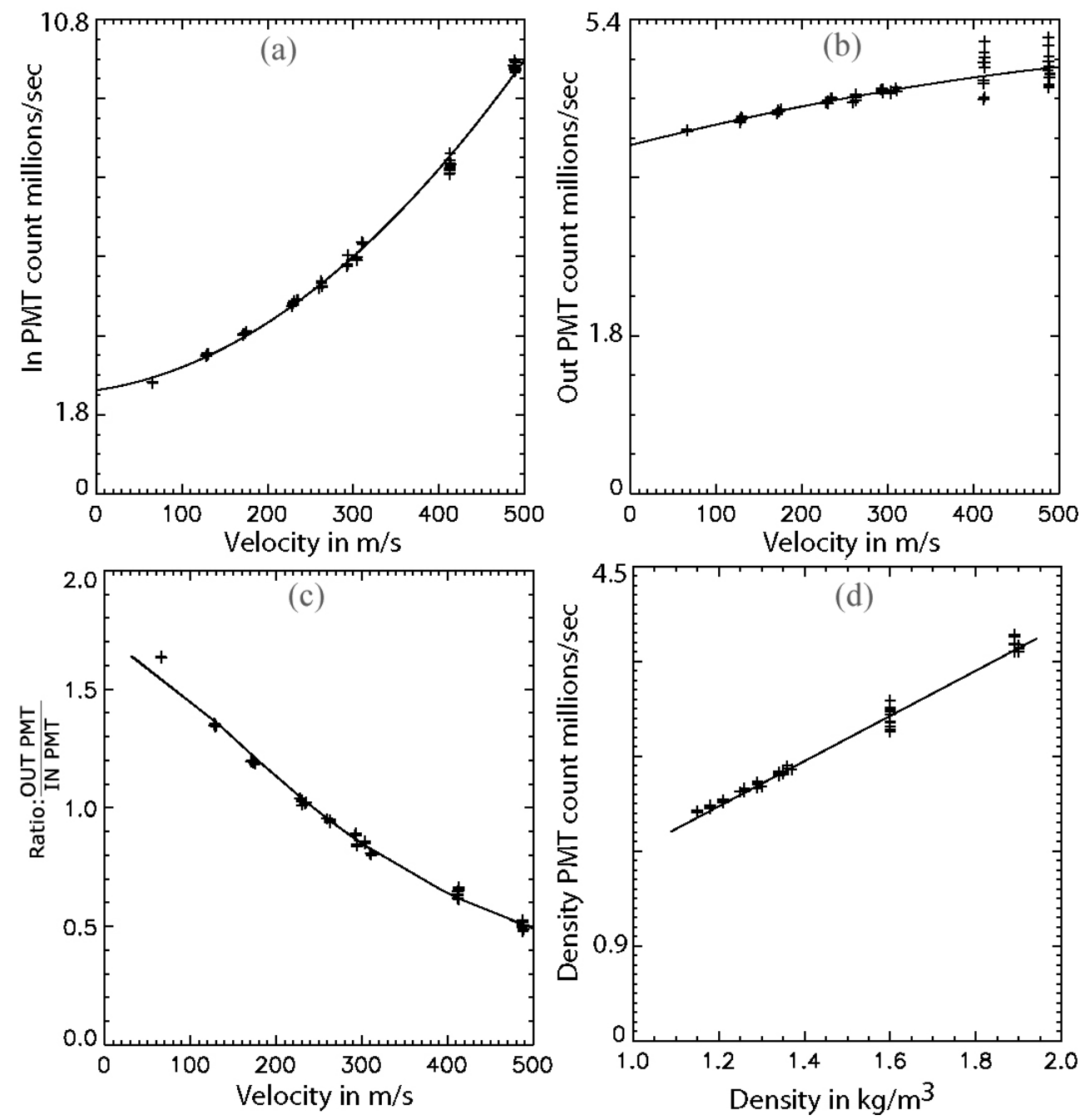

Fig 7. Density and velocity calibration using 3 photo-multiplier tubes.
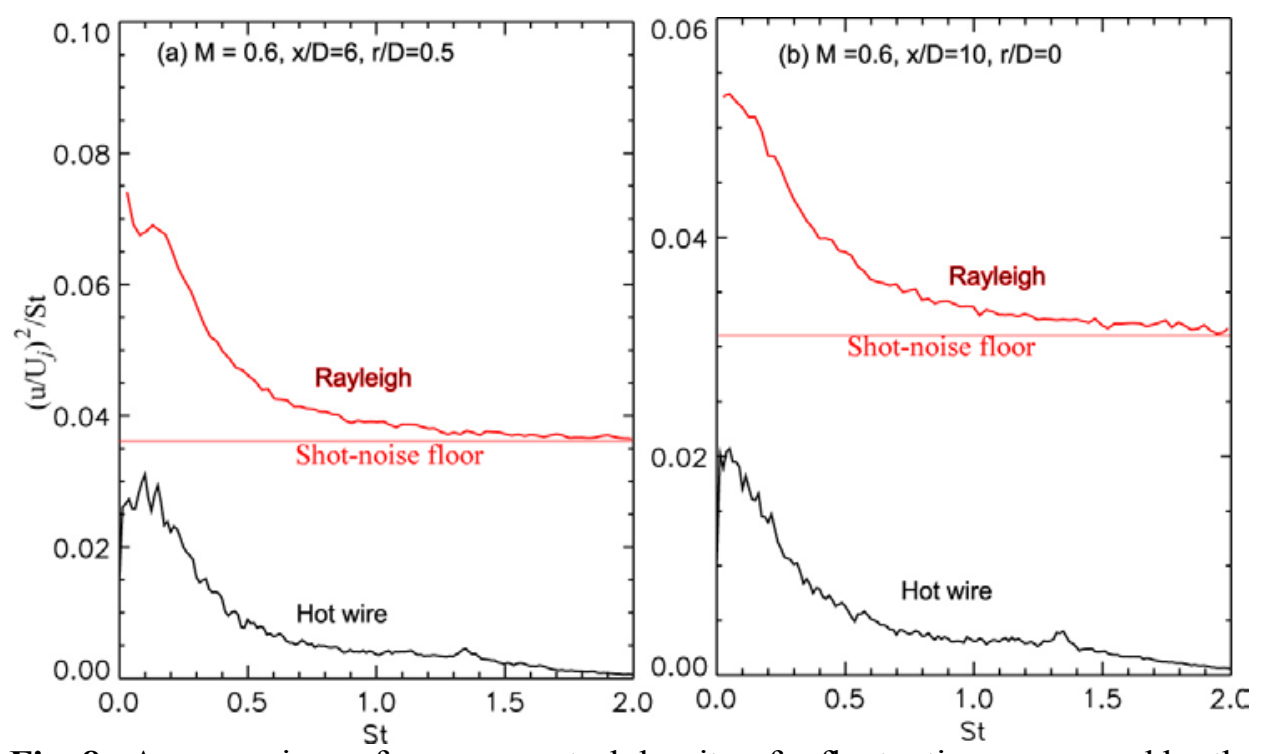

Fig 8. A comparison of power spectral density of u fluctuations measured by the Rayleigh technique and a hot-wire probe at indicated locations of Mach 0.6 plume. 

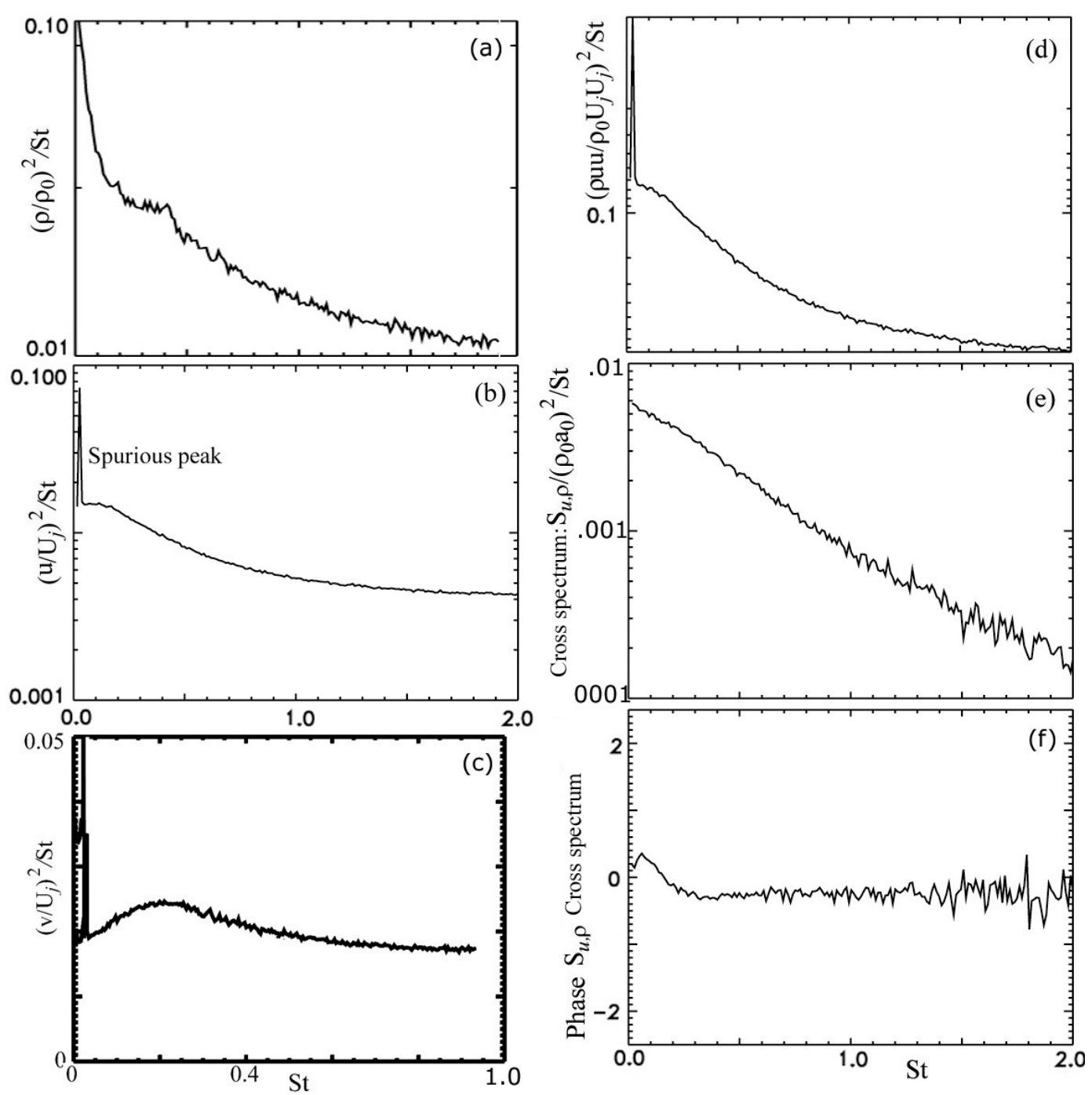

Fig 9. Power spectral density of (a) air density, (b) axial velocity, (c) radial velocity, (d) density*(axial velocity) ${ }^{2}$ fluctuations; (e) density-axial velocity cross-spectrum, and (f) phase of cross-spectrum in Mach 1.4 jet at centerline \& $\mathrm{x} / \mathrm{D}=10$ 

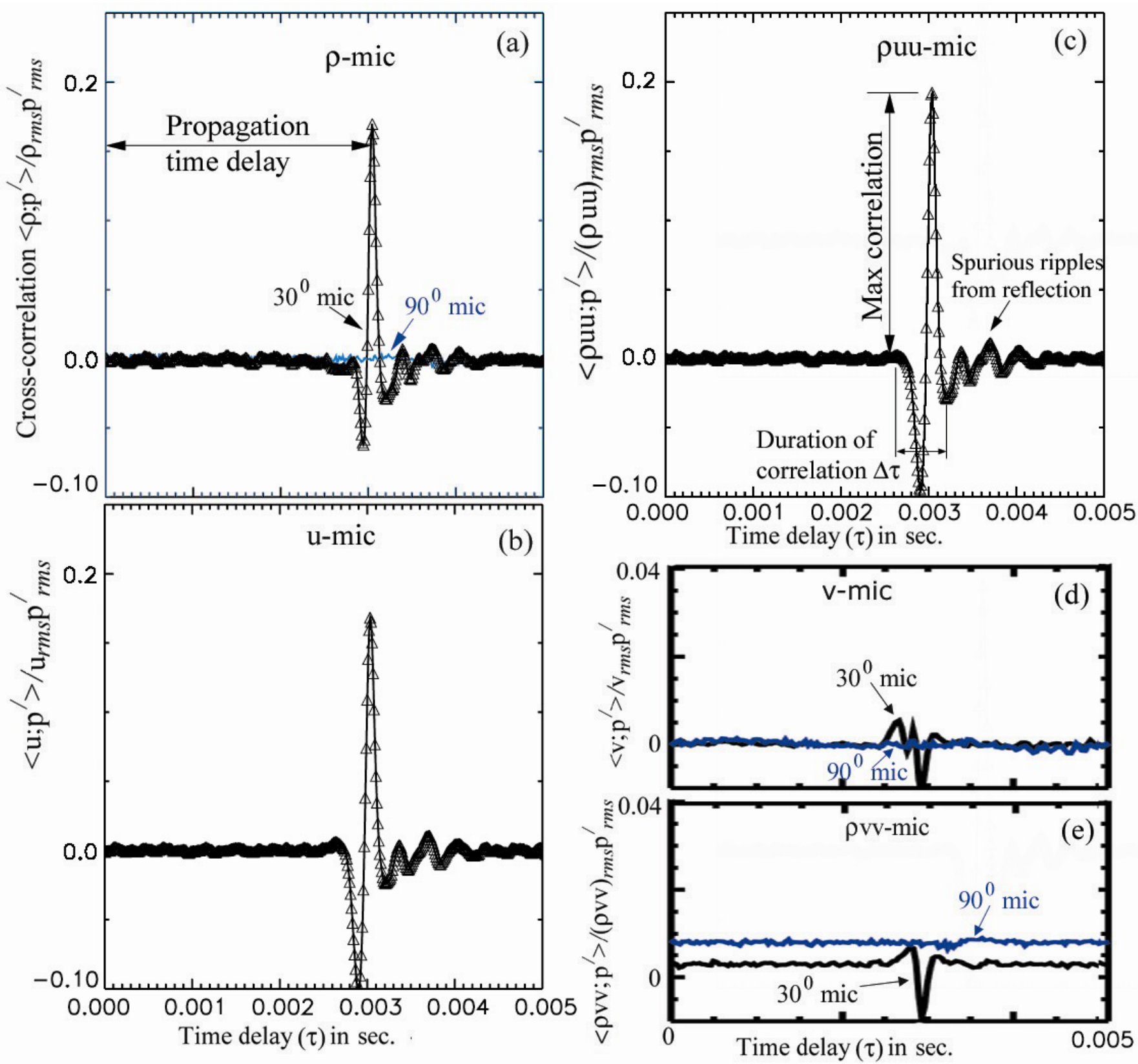

Fig 10. Normalized cross-correlation between sound pressure fluctuations $p^{\prime}$ and (a) air density $\rho$, (b) puu, (c) axial velocity $u,(d)$ radial velocity $v \&(e) \rho v v$ fluctuations measured at $x / D=10$ and centerline of $M=1.4$ jet. The microphone was kept at $50 \mathrm{D}$ and $30^{\circ}$ to jet axis unless otherwise mentioned. 

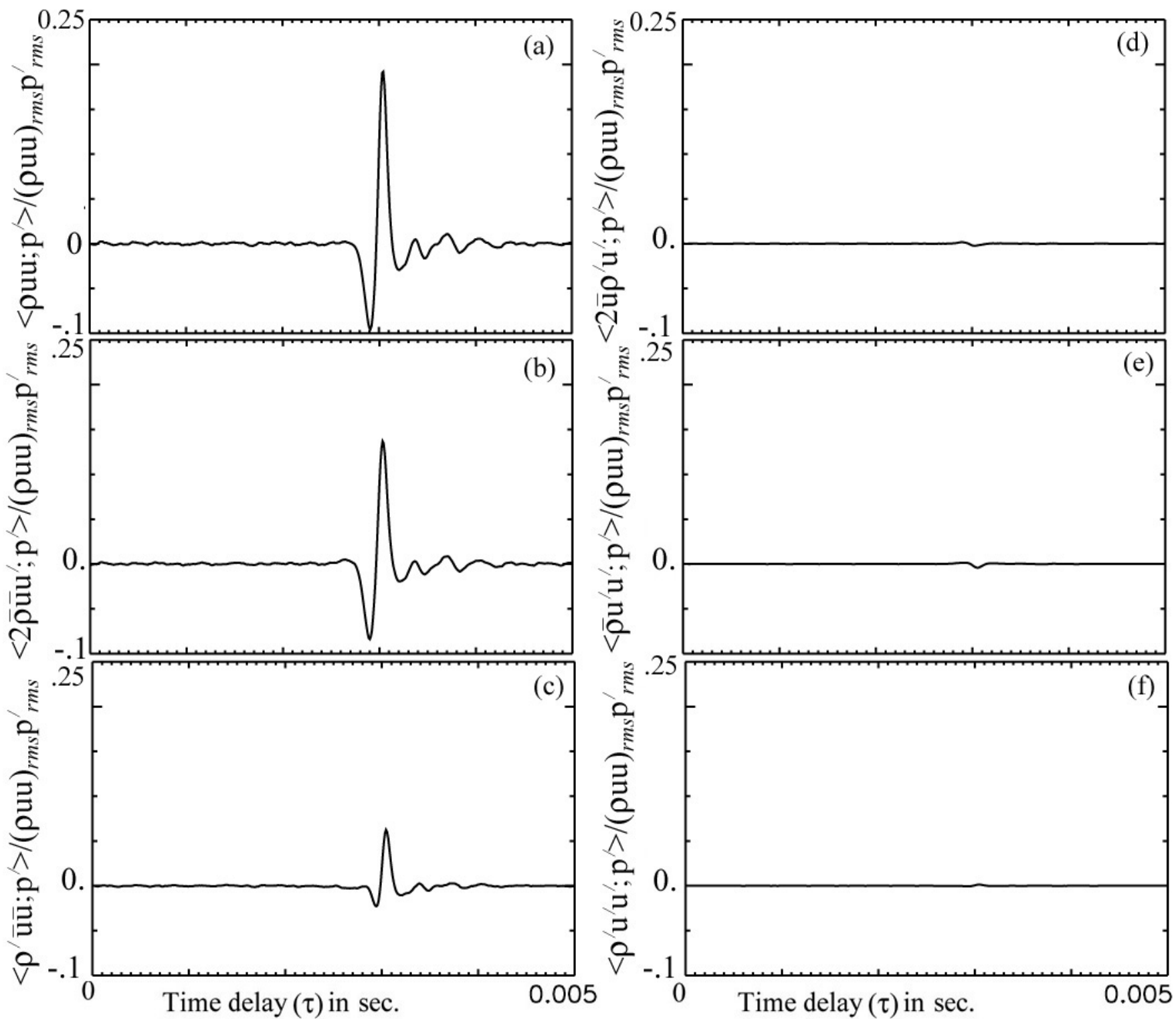

Fig. 11. Normalized cross-correlation between sound pressure fluctuations and (a) puu fluctuations (b)-(f) various Reynolds decomposed terms of puu measured at $\mathrm{x} / \mathrm{D}=10$ and centerline of $\mathrm{M}=1.4$ jet. The microphone was kept at 50D and $30^{\circ}$ to jet axis. 


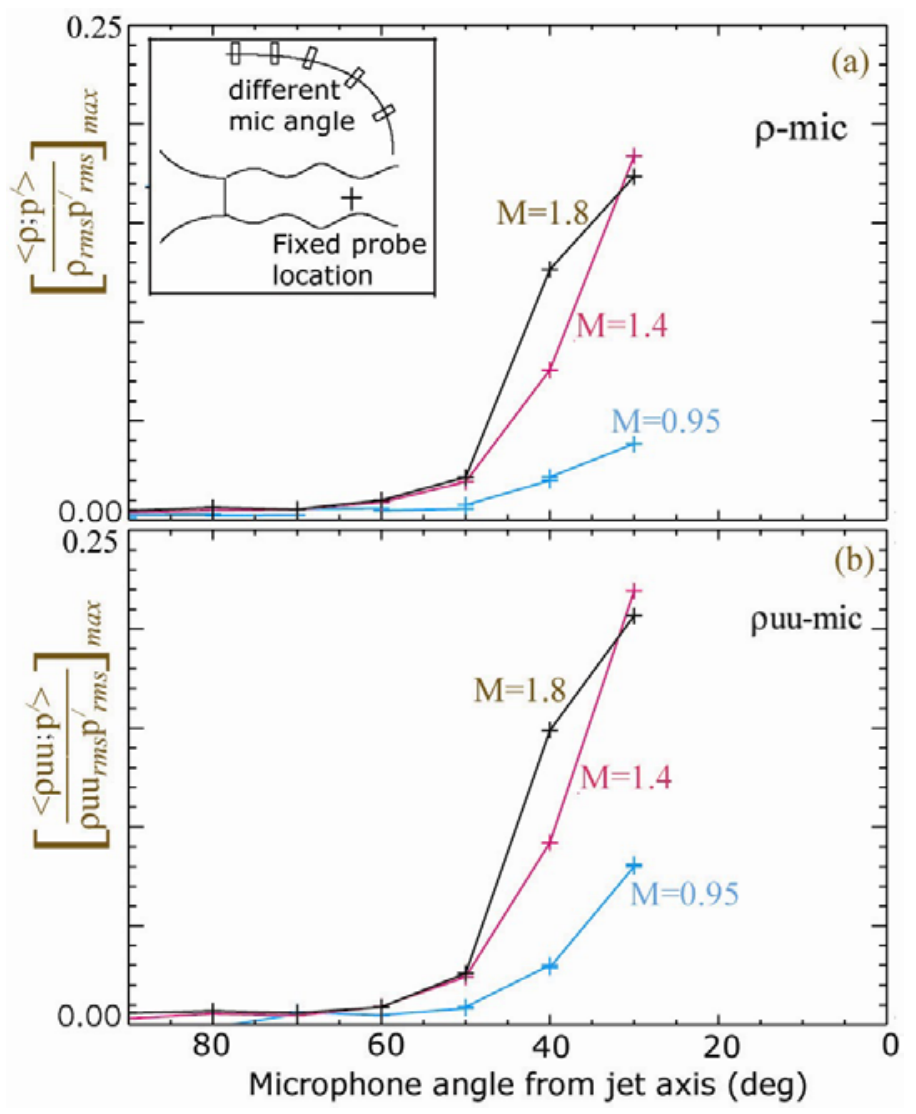

Fig. 12. Angular dependence of peak correlation between microphone pressures and (a) $\rho$, (b) puu fluctuations. The laser probe was kept fixed at centerline \& $\mathrm{x} / \mathrm{D}=8(\mathrm{M}=0.95$ case $)$ or $10(\mathrm{M}=1.4)$, or $12(\mathrm{M}=1.8)$ while the microphone was moved at various angular locations on a $51 \mathrm{D}$ arc.

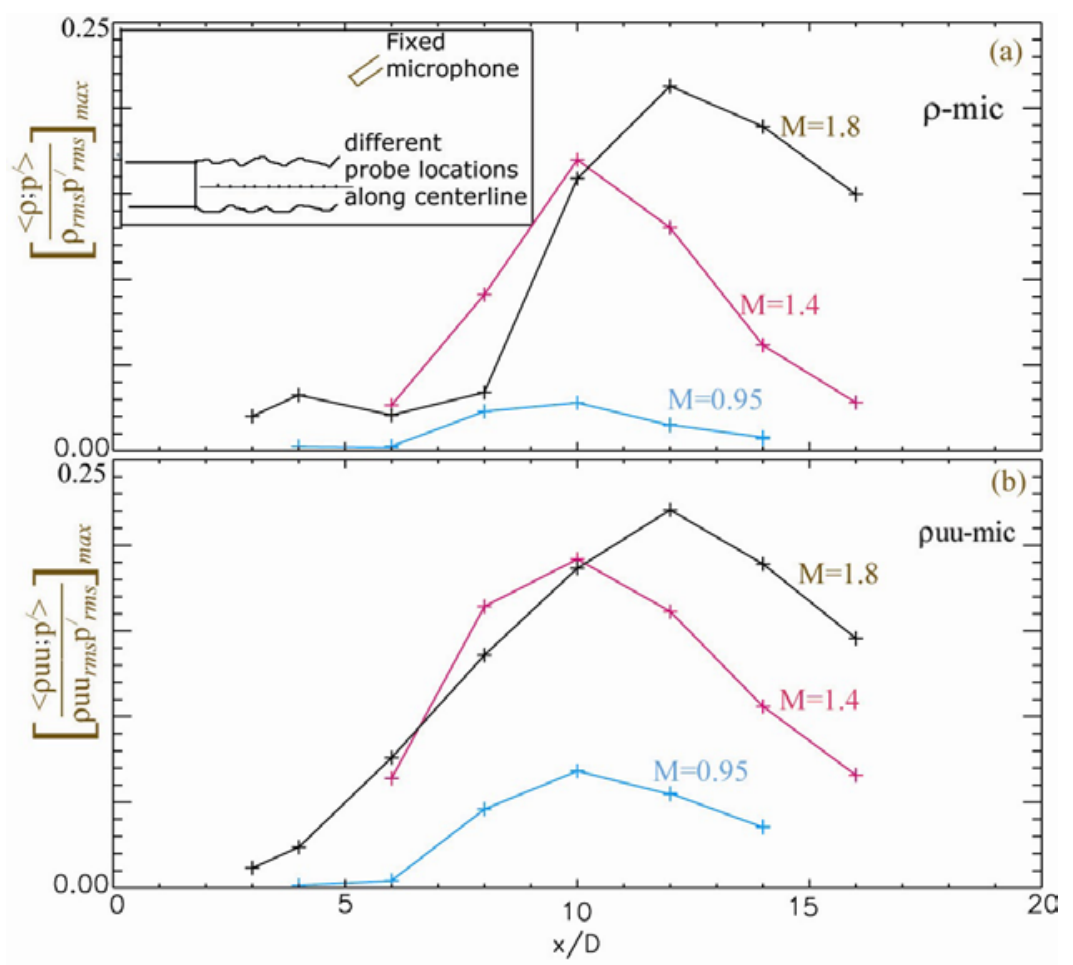

Fig. 13. Axial dependence of peak correlation between microphone pressures and (a) $\rho$, (b) puu fluctuations measured along centerline of indicated Mach number plumes. Microphone was kept fixed at 50D and $30^{\circ}$ to jet axis. 


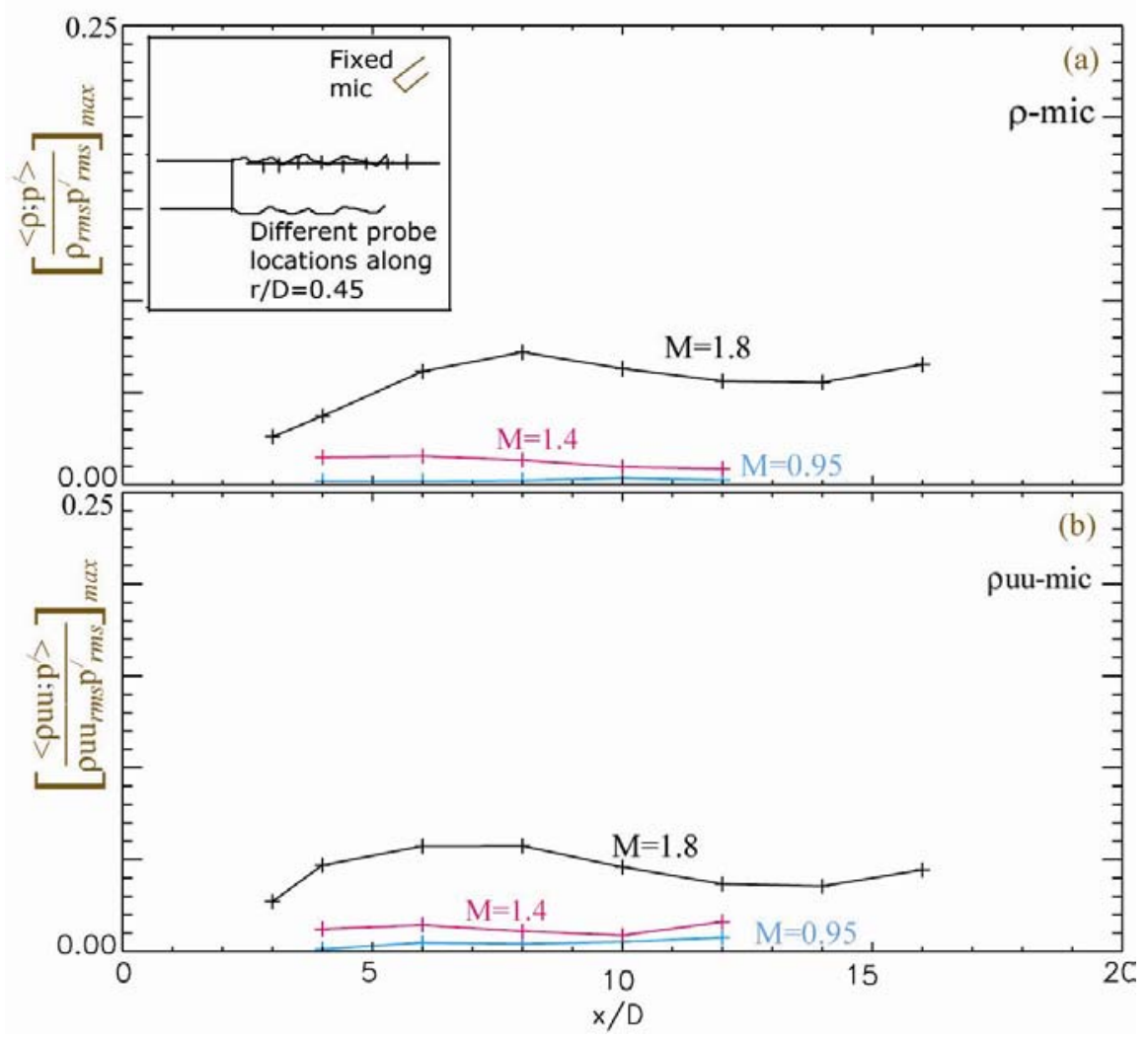

Fig. 14. Axial dependence of peak correlation between microphone pressures and (a) $\rho$, (b) puu fluctuations measured along shear layer $(r / D=0.45)$ of indicated Mach number plumes. Microphone was kept fixed at 50D and $30^{\circ}$ to jet axis.

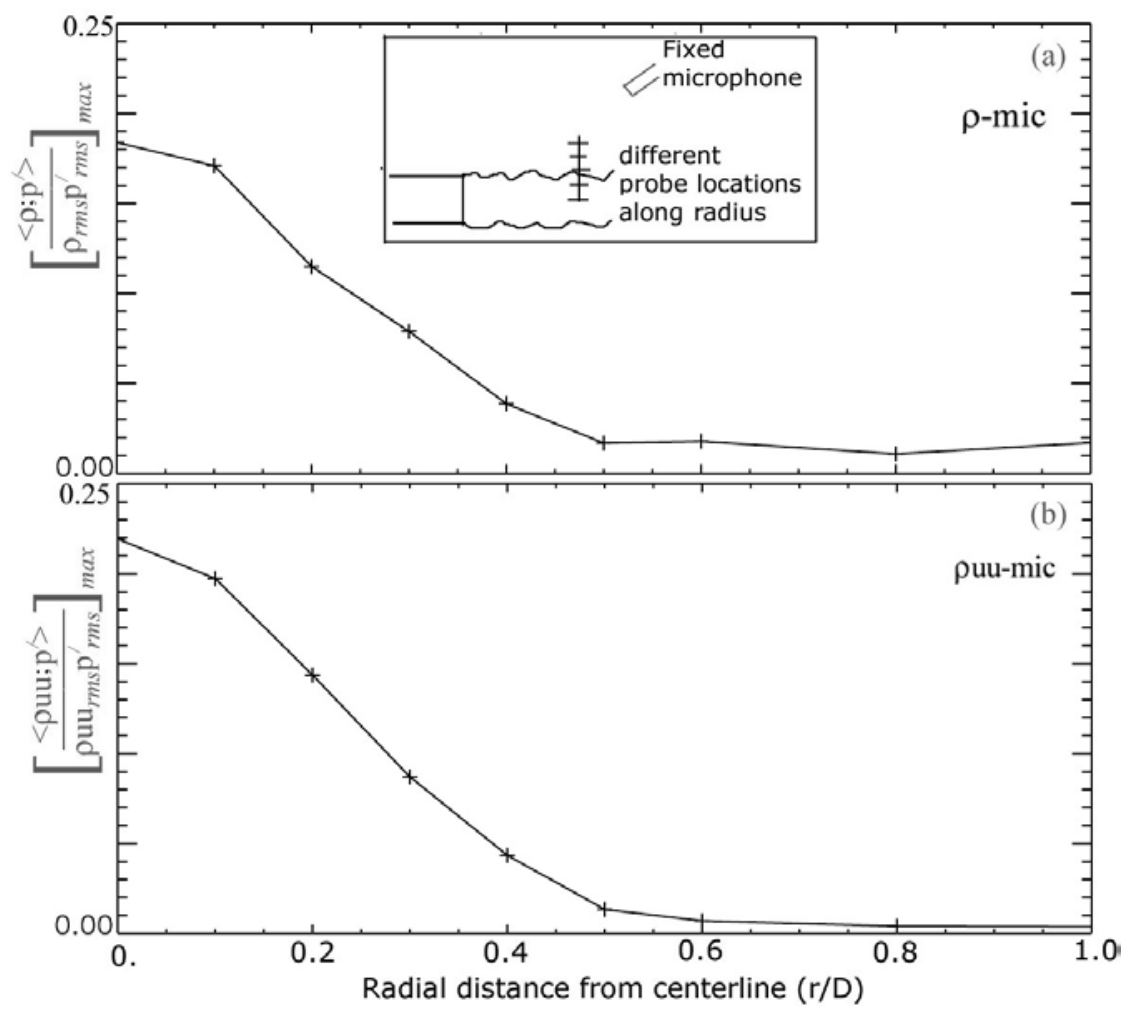

Fig. 15. Radial dependence of peak correlation between microphone pressures and (a) $\rho$, (b) puu fluctuations, measured at $\mathrm{x} / \mathrm{D}=10$ and various radial positions, in Mach 1.4 plume. Microphone was kept fixed at $50 \mathrm{D}$ and $30^{\circ}$ to jet axis. 

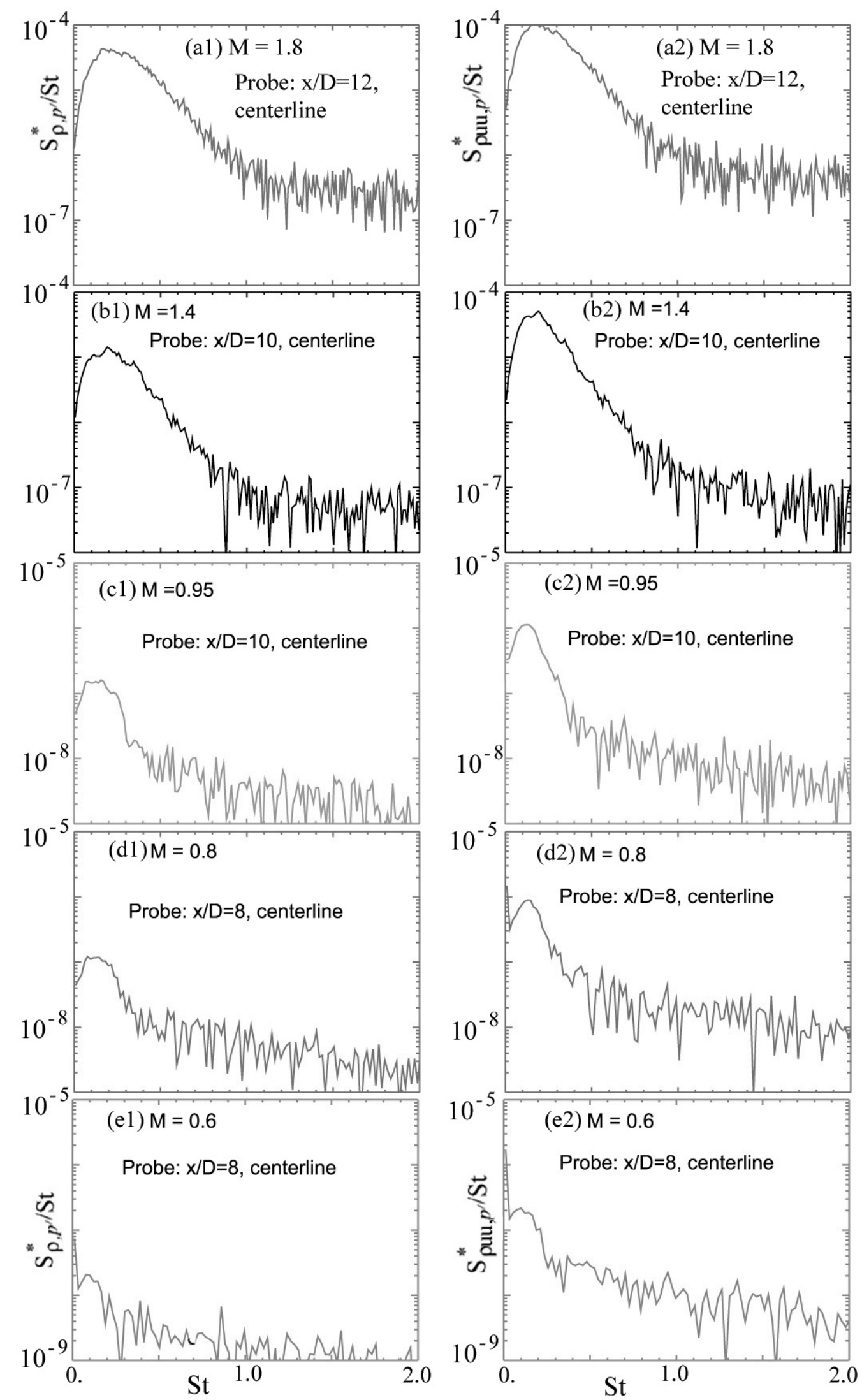

Fig. 16. Normalized cross-spectrum of sound pressure fluctuations $p^{\prime}$ and (a1 to e1) air density fluctuations, (a2 to e2) puu fluctuations from indicated probe locations in various Mach number plumes. Microphone was kept at $30^{\circ}$ and 50D away. 

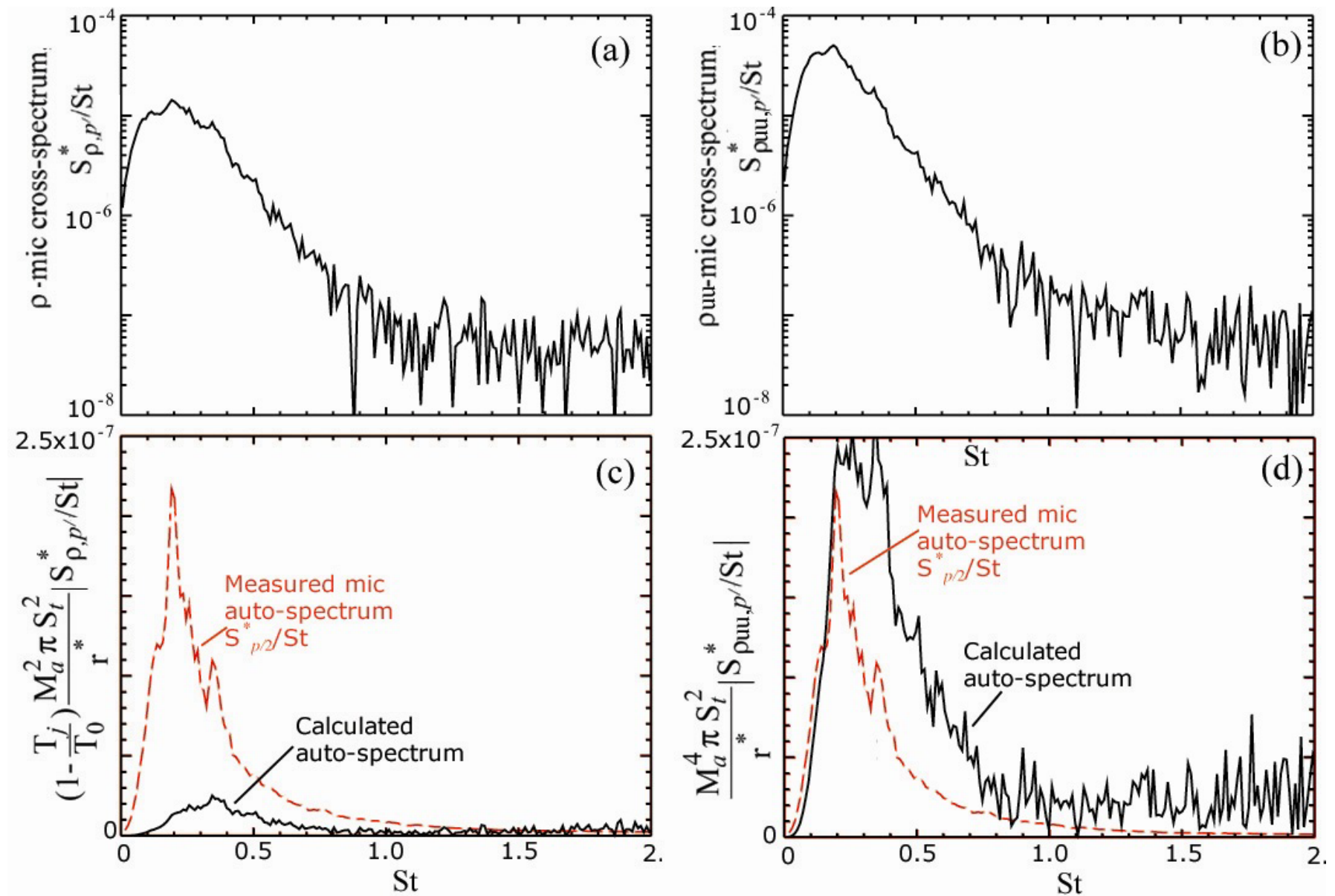

Fig. 17. Relative contribution to the $30^{\circ}$-microphone auto-spectrum from $\rho v v-p^{\prime}$ and $\rho-p^{\prime}$ cross-spectra measured in Mach 1.4 plume; probe location $\mathrm{x} / \mathrm{D}=10$ \& centerline. (a) $\rho$-p cross-spectra, (b) $\rho v v-p$ cross-spectra. Calculated contribution from (c) dipole (equation 14), and (d) quadrupole (equation 13) terms.

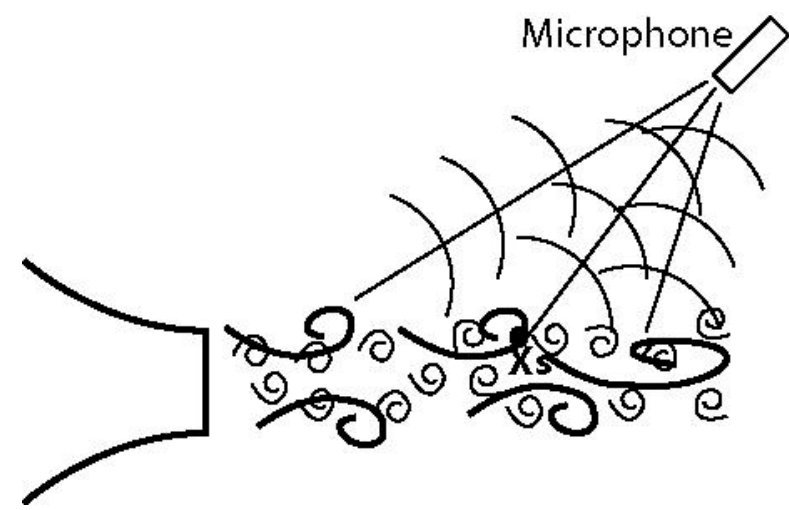

Fig. 18. Schematic of large and small scale turbulent structures and noise radiation. 

Public reporting burden for this collection of information is estimated to average 1 hour per response, including the time for reviewing instructions, searching existing data sources, gathering and maintaining the data needed, and completing and reviewing the collection of information. Send comments regarding this burden estimate or any other aspect of this collection of information, including suggestions for reducing this burden, to Washington Headquarters Services, Directorate for Information Operations and Reports, 1215 Jefferson Davis Highway, Suite 1204, Arlington, VA 22202-4302, and to the Office of Management and Budget, Paperwork Reduction Project (0704-0188), Washington, DC 20503.

\begin{tabular}{|l|l|l|}
\hline 1. AGENCY USE ONLY (Leave blank) & $\begin{array}{c}\text { 2. REPORT DATE } \\
\text { May } 2004\end{array}$ & $\begin{array}{r}\text { 3. REPORT TYPE AND DATES COVERED } \\
\text { Technical Memorandum }\end{array}$ \\
\hline
\end{tabular}

4. TITLE AND SUBTITLE 5. FUNDING NUMBERS

Further Progress in Noise Source Identification in High Speed Jets via Causality Principle

\section{AUTHOR(S)}

WBS-22-714-08-14

J. Panda, R.G. Seasholtz, and K.A. Elam

7. PERFORMING ORGANIZATION NAME(S) AND ADDRESS(ES)

National Aeronautics and Space Administration

John H. Glenn Research Center at Lewis Field

Cleveland, Ohio 44135-3191
8. PERFORMING ORGANIZATION REPORT NUMBER

E-13963

\section{SPONSORING/MONITORING AGENCY NAME(S) AND ADDRESS(ES)}

National Aeronautics and Space Administration

Washington, DC 20546-0001
10. SPONSORING/MONITORING AGENCY REPORT NUMBER

NASA TM-2004-212385

AIAA-2003-3126

\section{SUPPLEMENTARY NOTES}

Prepared for the Nineth Aeroacoustics Conference and Exhibit cosponsored by the American Institute of Aeronautics and Astronautics and the Confederation of European Aerospace Societies, Hilton Head, South Carolina, May 12-14, 2003. J. Panda, Ohio Aerospace Institute, Brook Park, Ohio 44142; R.G. Seasholtz, NASA Glenn Research Center; K.A. Elam, Akima Corporation, Fairview Park, Ohio 44126. Responsible person, J. Panda, organization code 5940, 216-433-8891.

12a. DISTRIBUTION/AVAILABILITY STATEMENT

\section{2b. DISTRIBUTION CODE}

Unclassified - Unlimited

Subject Category: 45

Distribution: Nonstandard

Available electronically at http://gltrs.grc.nasa.gov

This publication is available from the NASA Center for AeroSpace Information, 301-621-0390.

\section{ABSTRACT (Maximum 200 words)}

To locate noise sources in high-speed jets, the sound pressure fluctuations $\mathrm{p}^{\prime}$, measured at far field locations, were correlated with each of density $\rho$, axial velocity $\mathrm{u}$, radial velocity $\mathrm{v}$, $\rho u$ and $\rho \mathrm{vv}$ fluctuations measured from various points in fully expanded, unheated plumes of Mach number $0.95,1.4$ and 1.8. The velocity and density fluctuations were measured simultaneously using a recently developed, non-intrusive, point measurement technique based on molecular Rayleigh scattering (Seasholtz, Panda, and Elam, AIAA Paper 2002-0827). The technique uses a continuous wave, narrow line-width laser, Fabry-Perot interferometer and photon counting electronics. The far field sound pressure fluctuations at $30^{\circ}$ to the jet axis provided the highest correlation coefficients with all flow variables. The correlation coefficients decreased sharply with increased microphone polar angle, and beyond about $60^{\circ}$ all correlation mostly fell below the experimental noise floor. Among all correlations $<\rho u u ; p^{\prime}>$ showed the highest values. Interestingly, $<\rho ; p^{\prime}>$, in all respects, were very similar to $\left\langle\rho \mathrm{uu} ; \mathrm{p}^{\prime}>\right.$. The $\left\langle\mathrm{v} ; \mathrm{p}^{\prime}>\right.$ and $\left\langle\rho \mathrm{vv}\right.$; $\mathrm{p}^{\prime}>$ correlations with $90^{\circ}$ microphone fell below the noise floor. By moving the laser probe at various locations in the jet it was found that the strongest noise source lies downstream of the end of the potential core and extends many diameters beyond. Correlation measurement from the lip shear layer showed a Mach number dependency. While significant correlations were measured in Mach 1.8 jet, values were mostly below the noise floor for subsonic Mach 0.95 jet. Various additional analyses showed that fluctuations from large organized structures mostly contributed to the measured correlation, while that from small scale structures fell below the noise floor.

\section{SUBJECT TERMS}

Rayleigh scattering; Jet aircraft noise

15. NUMBER OF PAGES

32

16. PRICE CODE

\begin{tabular}{|c|c|c|}
\hline $\begin{array}{c}\text { 17. SECURITY CLASSIFICATION } \\
\text { OF REPORT } \\
\text { Unclassified }\end{array}$ & $\begin{array}{c}\text { 18. SECURITY CLASSIFICATION } \\
\text { OF THIS PAGE } \\
\text { Unclassified }\end{array}$ & $\begin{array}{c}\text { 19. SECURITY CLASSIFICATION } \\
\text { OF ABSTRACT } \\
\text { Unclassified }\end{array}$ \\
\hline
\end{tabular}

NSN 7540-01-280-5500

Standard Form 298 (Rev. 2-89) 

